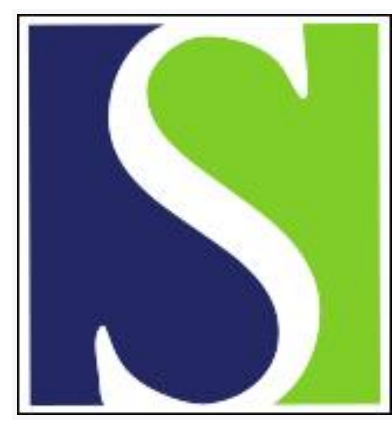

Scand J Work Environ Health 2009;35(4):261-281

https://doi.org/10.5271/sjweh.1337

Issue date: 31 Jul 2009

Common prognostic factors of work disability among employees with a chronic somatic disease: a systematic review of cohort studies

by Detaille SI, Heerkens YF, Engels JA, van der Gulden JWJ, van Dijk $\mathrm{FJH}$

Affiliation: HAN University of Applied Sciences, Verlengde Groenestraat 75, PO box 6960, 6503 GL Nijmegen, the Netherlands. sarah.detaille@han.nl

Refers to the following texts of the Journal: 2009;35(4):282-283 2003;29(2):134-142

The following articles refer to this text: 2009;35(6):479; 2010;36(6):473-483; 2011;37(5):402-410; 2020;46(4):402-409

Key terms: chronic disease; chronic somatic disease; cohort study; employee; occupational health; prognostic factor; review; risk factor; self-management; somatic disease; systematic review; work disability

This article in PubMed: www.ncbi.nlm.nih.gov/pubmed/19562236 


\title{
Common prognostic factors of work disability among employees with a chronic somatic disease: a systematic review of cohort studies
}

\author{
by Sarah I Detaille, MA, ${ }^{1}$ Yvonne F Heerkens, PhD, ${ }^{2}$ Josephine A Engels, PhD, ${ }^{3}$ Joost WJ van der Gulden, \\ PhD, ${ }^{4}$ Frank JH van Dijk, PhD ${ }^{5}$
}

\begin{abstract}
Detaille SI, Heerkens YF, Engels JA, van der Gulden JWJ, van Dijk FJH. Common prognostic factors of work disability among employees with a chronic somatic disease: a systematic review of cohort studies. Scand J Work Environ Health. 2009;35(4):261-281.
\end{abstract}

\begin{abstract}
Objective Based on prospective and retrospective disease cohort studies, the aim of this review was to determine common prognostic factors for work disability among employees with rheumatoid arthritis, asthma, chronic obstructive pulmonary disease, diabetes mellitus, and ischemic heart disease (IHD).

Methods A systematic literature search in Medline (1990-2008) and Embase (1990-2008) was carried out to identify relevant cohort studies using a well-defined list of inclusion and quality criteria.

Results We identified 43 relevant cohort studies with sufficient methodological quality (20 for rheumatoid arthritis, 3 for asthma and 20 for IHD). The common prognostic factors for work disability found in all the diseases were: perceived health complaints, limitation in daily physical activities caused by the disease (high versus low), heavy manual work, and female gender. The common positive prognostic factors for rheumatoid arthritis and IHD were age (high versus low) and sickness absence. The common negative factors for rheumatoid arthritis and IHD were education (high versus low) and ethnic origin (white versus non-white).

Conclusions As many prognostic factors for work disability are similar for employees with various chronic diseases, it is possible to detect high risk groups. This information supports the development and implementation of a general disability management intervention for employees suffering from a chronic disease to overcome health-related limitations at work.
\end{abstract}

Key terms chronic disease; risk factor; self-management; occupational health.

The percentage of the working-age population with a long-standing health problem or disability (including non-specific low back pain and mental disorders) varies widely among countries in Europe, with the highest percentage (32.2\%) found in Finland and the lowest in Romania (5.8\%) (1). Over the next 20 years, prognostic studies predict an increase in the incidence of chronic diseases in the working population, such as rheumatoid arthritis, chronic obstructive pulmonary disease (COPD), asthma, diabetes mellitus, and ischemic heart disease (IHD), merely due to the aging of the working population (2-3). Despite improvements in facilities and medical care for people with a chronic medical condition, the presence of chronic disease is still a major cause of long-term sickness absence and job loss in Europe.
Labor force participation in Europe is much lower for disabled people. For individuals with a chronic medical condition between 16-64 years of age, the unemployment rate is nearly twice as high as nondisabled individuals. Only one in six employees with a long-standing health problem or disability, who face work restrictions in Europe, is provided some assistance to work (1).

In the Netherlands, only one third of the people between 16-64 years of age with a chronic disease have a paid job in comparison to two thirds of the general population. Of those employees who have a chronic disease, $30 \%$ experience problems at the workplace related to the disease (4). The unemployment rate among the disabled is higher than that of the non-disabled for

1 Seneca, Expertise Centre for Sport, Work and Health, HAN University of Applied Sciences, Nijmegen, the Netherlands.

2 HAN University of Applied Sciences, Nijmegen, the Netherlands.

3 HAN University of Applied Sciences, Nijmegen, the Netherlands.

4 Department of Occupational Health, Radboud University Nijmegen Medical Centre, Nijmegen, the Netherlands.

5 Coronel Institute of Occupational Health, Academic Medical Center, University of Amsterdam, Amsterdam, the Netherlands.

Correspondence to: Sarah I Detaille, HAN University of Applied Sciences, Verlengde Groenestraat 75, PO box 6960, 6503 GL Nijmegen, the Netherlands. [E-mail: sarah.detaille@han.nl] 
various reasons. One reason is probably the "discouraged worker" effect. Many disabled persons may believe that they are very unlikely to get a job, so they do not even attempt to find one and are, therefore, classified as inactive (5).

In studies of work disability due to rheumatoid arthritis, a relation has been found between becoming work disabled and variables such as: demanding physical work, low education, increasing age ( $>50$ years), pain and self-reported functional status measured with the (modified) Health Assessment Questionnaire (HAQ), disease duration, and motivation to work (6-8). In studies on work disability due to asthma, a relation was found between work factors, education, general health, and motivation to work (9-11). Until now, no reviews have systematically evaluated the correspondence between prognostic factors of work disability among employees with a variety of chronic somatic diseases.

In a qualitative (concept mapping) study to explore the prerequisites for employees with a chronic somatic disease to function at work, working with employees and health professionals, we found relatively more disease-generic (ie, common) than disease-specific factors to enable employees to cope. These included: self-acceptance, self-efficacy, support from management and colleagues, and professional advice (12). It is important to search for evidence of common factors as this knowledge can be used to train health professionals in occupational and general health to become aware of employees who have an increased risk of becoming work disabled. Consequently, knowledge about these factors is needed to develop and implement evidencebased interventions to support patients/workers not only in retaining their jobs, but also for the purposes of rehabilitation and return-to-work programs. It might be postulated that many employees with a chronic disease (with the exception of specific work-related chronic diseases caused by occupational exposure) could continue working if they received support in coping with their disease at work. Such employees should be facilitated to continue any form of work participation. This includes the option to change jobs if the employee is not able to continue in his or her current job due to a diseaserelated limitation or continued occupational exposure. Occupational health services, primary health care, medical specialists, nurses, and allied health personnel can improve the quality of care by adjusting their actions to the needs of specific risk groups.

The objective of this study was to search for prognostic factors of work disability which are common to various chronic somatic diseases. A prognostic factor is a risk factor for becoming work disabled, which - when identified - can be used in training health professionals to recognize and support employees who are at risk. The identification of such prognostic factors also enables the development and implementation of disability management interventions.

In this systematic review, we focused on rheumatoid arthritis, COPD, asthma, diabetes mellitus, and IHD as these diseases have a high prevalence in the Netherlands and in other countries (13). Our hypothesis was that several common personal, work- and disease-related factors predict work disability for a variety of chronic diseases. For the purpose of this study, we selected a number of frequently occurring chronic somatic diseases representing a wide variety of diseases in an effort to look for common prognostic factors.

\section{Methods}

\section{Search strategy}

In January 2007, with a follow-up in October 2008, we conducted a search in Medline (1990-2008) and Embase (1990-2008) using the following search terms both as MeSH term and text word in the title and abstract: (i) rheumatoid arthritis or diabetes mellitus or COPD or asthma or myocardial ischemia; and (ii) occupation or participation or sustainability or employment or unemployment or workplace or career mobility or rehabilitation, vocational or vocation or work or working or labor or job or jobs; and (iii) ability or abilities or disability or disabilities or return to work or continuing to work or functioning or performance or participation or work capacity or sick leave or absenteeism or vocation/disability or disability evaluation; and (iv) prognostic factors or predictive factors. The search strategy was formulated in NCBI (Medline) and was adapted for use in Webspirs (Embase). A combination of the words from (i), (ii), (iii), and (iv) above was used and, as limitation, only English publications were selected.

\section{Selection and methodological quality assessment}

Two reviewers independently selected relevant abstracts from the articles retrieved from the search strategy. If abstracts provided insufficient information, the full text of each article was used. We applied two sets of criteria: the first set to select the study population and type of study, and the second set to screen the articles on quality. In the first screening round, studies were selected if they met all of the following criteria: (i) the study population consisted of subjects with rheumatoid arthritis, COPD, asthma, diabetes mellitus or IHD; (ii) the chronic diseases were not work-related (caused by work); (iii) the study examined work disability, return to work, or continuing to work as the outcome; (iv) the subjects were 18-65 years old (working population); (v) the subjects were employed at the start of the study; 
(vi) the subjects were not fully work disabled and had not been absent from work for more than two years at the start of the study; (vii) the study must have been a cohort study (prospective/retrospective); and (viii) the study must have applied an appropriate statistical model (univariate or if available multivariate).

Following a reading of the abstract, the authors decided either to include or exclude each study; in some cases further discussion was needed to address whether a study should be included (indecisive result). Disagreements regarding inclusion status were resolved by consensus. When no consensus was reached, the abstract or full text was screened by a third reviewer who then decided.The methodological quality of the selected studies was assessed using the criteria list of the Dutch Cochrane Centre for cohort studies (14) which is set out in table 1. Based on the aforementioned eight criteria, the studies were classified as being of "high

Table 1. Background information and quality criteria for the selected cohort studies. $[P / R=$ prospective and retrospective study, $\mathrm{P}=$ prospective study, $\mathrm{HQ}=$ high quality (meets $\geq 7$ criteria), $\mathrm{MQ}=$ medium quality (meets $5-6$ criteria), $\mathrm{LQ}=$ low quality (meets $<5$ criteria)]

\begin{tabular}{|c|c|c|c|c|c|c|c|c|c|c|c|c|c|}
\hline Studies & Design & $\begin{array}{l}\text { Sample } \\
\text { size }\end{array}$ & $\begin{array}{l}\text { Duration } \\
\text { of follow } \\
\text { up in } \\
\text { years }\end{array}$ & $\begin{array}{l}\text { Study } \\
\text { popula- } \\
\text { tion fully } \\
\text { described }\end{array}$ & $\begin{array}{l}\text { Selec- } \\
\text { tion bias } \\
\text { can be } \\
\text { excluded }\end{array}$ & $\begin{array}{l}\text { Prognostic } \\
\text { factor } \\
\text { described } \\
\& \text { method } \\
\text { correctly } \\
\text { described? }\end{array}$ & $\begin{array}{l}\text { Outcome } \\
\text { assess- } \\
\text { ment (work } \\
\text { disability) } \\
\text { \& method } \\
\text { correctly } \\
\text { described? }\end{array}$ & $\begin{array}{l}\text { Outcome } \\
\text { assess- } \\
\text { ment } \\
\text { blinded } \\
\text { to prog- } \\
\text { nostic } \\
\text { factor? }\end{array}$ & $\begin{array}{l}\text { Follow } \\
\text { up of } \\
\text { patients } \\
(>1 \\
\text { year) }\end{array}$ & $\begin{array}{l}\text { Selected } \\
\text { follow up } \\
\text { loss be } \\
\text { excluded }\end{array}$ & $\begin{array}{l}\text { Appro- } \\
\text { priate } \\
\text { design } \\
\text { used a }^{\text {a }}\end{array}$ & $\begin{array}{l}\text { Total } \\
\text { quality } \\
\text { score } \\
(0-8)\end{array}$ & $\begin{array}{l}\text { Quality } \\
\text { label }\end{array}$ \\
\hline
\end{tabular}

Cohort studies for employees with rheumatoid arthritis

\begin{tabular}{|c|c|c|c|c|c|c|c|c|c|c|c|c|c|}
\hline $\begin{array}{l}\text { Albers } \\
\text { et al (38) }\end{array}$ & $\mathrm{P} / \mathrm{R}$ & 186 & 3 & + & + & + & + & + & + & - & + & 7 & $\mathrm{HQ}$ \\
\hline $\begin{array}{l}\text { Barrett } \\
\text { et al (39) }\end{array}$ & $P$ & $\begin{array}{l}110- \\
160\end{array}$ & $4.1-8.6$ & + & + & + & + & + & + & - & + & 7 & $\mathrm{HQ}$ \\
\hline $\begin{array}{l}\text { Borg } \\
\text { et al (43) }\end{array}$ & $P$ & 83 & 2 & + & + & + & + & + & + & & - & 7 & $\mathrm{HQ}$ \\
\hline $\begin{array}{l}\text { Chung } \\
\text { et al (25) }\end{array}$ & $P$ & 633 & $>3$ & + & - & + & + & + & + & & + & 7 & $\mathrm{HQ}$ \\
\hline $\begin{array}{l}\text { Eberhardt } \\
\& \text { Fex (44) }\end{array}$ & $P$ & 106 & 4 & + & - & + & + & + & + & - & + & 6 & $\mathrm{MQ}$ \\
\hline $\begin{array}{l}\text { Fex } \\
\text { et al (42) }\end{array}$ & $P$ & 84 & 2 & + & - & + & + & + & + & & - & 6 & $\mathrm{MQ}$ \\
\hline $\begin{array}{l}\text { Holte } \\
\text { et al (57) }\end{array}$ & $P$ & 3316 & 2 & + & + & + & + & + & + & - & + & 7 & $\mathrm{HQ}$ \\
\hline $\begin{array}{l}\text { Jäntti } \\
\text { et al (55) }\end{array}$ & $P$ & 103 & $1-20$ & + & - & + & + & + & + & - & + & 6 & $\mathrm{MQ}$ \\
\hline $\begin{array}{l}\text { Mancuso } \\
\text { et al (8) }\end{array}$ & $P$ & 122 & 1 & + & - & + & + & + & - & & + & 6 & $\mathrm{MQ}$ \\
\hline $\begin{array}{l}\text { Mau } \\
\text { et al (35) }\end{array}$ & $P$ & 73 & 6 & + & - & + & + & + & + & & - & 6 & $\mathrm{MQ}$ \\
\hline $\begin{array}{l}\text { Odegaard } \\
\text { et al (54) }\end{array}$ & $P$ & 159 & 7 & + & + & + & + & + & + & - & + & 7 & $\mathrm{HQ}$ \\
\hline $\begin{array}{l}\text { Puolakka } \\
\text { et al (24) }\end{array}$ & $P$ & 162 & 5 & + & + & + & + & + & + & & + & 8 & $\mathrm{HQ}$ \\
\hline $\begin{array}{l}\text { Puolakka } \\
\text { et al (23) }\end{array}$ & $P$ & 162 & 5 & + & - & + & + & + & + & & + & 7 & $\mathrm{HQ}$ \\
\hline $\begin{array}{l}\text { Reisine } \\
\text { et al (22) }\end{array}$ & $P$ & 392 & 5 & + & - & + & + & + & + & - & + & 6 & $\mathrm{MQ}$ \\
\hline $\begin{array}{l}\text { Reisine } \\
\text { et al (7) }\end{array}$ & $P$ & 498 & 9 & + & - & + & + & + & + & - & - & 5 & $\mathrm{MQ}$ \\
\hline $\begin{array}{l}\text { Reisine } \\
\text { et al (41) }\end{array}$ & $P$ & 139 & .. & + & - & + & + & + & + & - & + & 6 & $\mathrm{HQ}$ \\
\hline $\begin{array}{l}\text { Sokka } \\
\text { et al (40) }\end{array}$ & $P$ & 82 & 10 & + & - & + & + & + & + & + & + & 7 & $\mathrm{HQ}$ \\
\hline $\begin{array}{l}\text { Straaton } \\
\text { et al (37) }\end{array}$ & $P$ & 218 & 1 & + & - & + & + & + & - & + & + & 6 & $\mathrm{MQ}$ \\
\hline $\begin{array}{l}\text { Wolfe \& } \\
\text { Hawley } \\
(36)\end{array}$ & $P$ & 823 & 8 & + & - & + & + & + & + & - & + & 6 & $\mathrm{MQ}$ \\
\hline $\begin{array}{l}\text { Young } \\
\text { et al (26) }\end{array}$ & P & 732 & 5 & + & - & + & + & + & + & + & $t$ & 7 & $\mathrm{HQ}$ \\
\hline
\end{tabular}


Table 1. Continued.

\begin{tabular}{|c|c|c|c|c|c|c|c|c|c|c|c|c|c|}
\hline Studies & Design & $\begin{array}{l}\text { Sample } \\
\text { size }\end{array}$ & $\begin{array}{l}\text { Duration } \\
\text { of follow } \\
\text { up in } \\
\text { years }\end{array}$ & $\begin{array}{l}\text { Study } \\
\text { popula- } \\
\text { tion fully } \\
\text { described }\end{array}$ & $\begin{array}{l}\text { Selec- } \\
\text { tion bias } \\
\text { can be } \\
\text { excluded }\end{array}$ & $\begin{array}{l}\text { Prognostic } \\
\text { factor } \\
\text { described } \\
\text { \& method } \\
\text { correctly } \\
\text { described? }\end{array}$ & $\begin{array}{l}\text { Outcome } \\
\text { assess- } \\
\text { ment (work } \\
\text { disability) } \\
\text { \& method } \\
\text { correctly } \\
\text { described? }\end{array}$ & $\begin{array}{l}\text { Outcome } \\
\text { assess- } \\
\text { ment } \\
\text { blinded } \\
\text { to prog- } \\
\text { nostic } \\
\text { factor? }\end{array}$ & $\begin{array}{l}\text { Follow } \\
\text { up of } \\
\text { patients } \\
(>1 \\
\text { year })\end{array}$ & $\begin{array}{l}\text { Selected } \\
\text { follow up } \\
\text { loss be } \\
\text { excluded }\end{array}$ & $\begin{array}{l}\text { Appro- } \\
\text { priate } \\
\text { design } \\
\text { used }^{\text {a }}\end{array}$ & $\begin{array}{l}\text { Total } \\
\text { quality } \\
\text { score } \\
(0-8)\end{array}$ & $\begin{array}{l}\text { Quality } \\
\text { label }\end{array}$ \\
\hline
\end{tabular}

\section{Cohort studies for employees with asthma}

Alexopoulos

\& Burdorf

(21)

Blanc
et al (10)

Yelin

et al (11)

$46 \quad 2$

$+$

\section{Cohort studies for employees with ischemic heart disease}

\begin{tabular}{|c|c|c|c|c|c|c|c|c|c|c|c|c|c|}
\hline $\begin{array}{l}\text { Abbas } \\
\text { et al (20) }\end{array}$ & $P$ & $450^{b}$ & 6 months & + & - & + & + & - & - & - & + & 4 & $\mathrm{LQ}$ \\
\hline $\begin{array}{l}\text { Abbott \& } \\
\text { Berry (46) }\end{array}$ & $P$ & $82^{c}$ & 1 & + & + & + & + & + & + & + & - & 7 & $H Q$ \\
\hline $\begin{array}{l}\text { Agren } \\
\text { et al (28) }\end{array}$ & $P$ & $25^{b}$ & 5 & + & + & + & + & + & + & + & - & 7 & $H Q$ \\
\hline $\begin{array}{l}\text { Bhatta- } \\
\text { charyya } \\
\text { et al, } 2007 \\
\text { (30) }\end{array}$ & $P$ & $126^{c}$ & 1 & + & + & + & + & + & + & + & + & 8 & $\mathrm{HQ}$ \\
\hline $\begin{array}{l}\text { Boudrez } \\
\text { et al (40) }\end{array}$ & $P$ & $530^{c}$ & 1 & + & + & + & + & + & + & + & + & 8 & $H Q$ \\
\hline $\begin{array}{l}\text { Boudrez \& } \\
\text { De Backer } \\
\text { (19) }\end{array}$ & $P$ & $\begin{array}{l}137^{b} \\
90^{c}\end{array}$ & 1 & + & + & + & + & + & + & + & + & 8 & $\mathrm{HQ}$ \\
\hline $\begin{array}{l}\text { Caine } \\
\text { et al (53) }\end{array}$ & $P$ & $100^{b}$ & 1 & + & + & + & + & + & + & + & + & 8 & $\mathrm{HQ}$ \\
\hline $\begin{array}{l}\text { Drory } \\
\text { et al (34) }\end{array}$ & $P$ & $833^{d}$ & 5 & + & + & + & + & + & + & + & + & 8 & $\mathrm{HQ}$ \\
\hline $\begin{array}{l}\text { Earle } \\
\text { et al (58) }\end{array}$ & $P$ & $289 c$ & 3 months & + & + & + & + & + & - & + & + & 7 & $\mathrm{HQ}$ \\
\hline $\begin{array}{l}\text { Engblom } \\
\text { et al (49) }\end{array}$ & $P$ & $201^{b}$ & 1 & + & + & + & + & + & + & + & + & 8 & $H Q$ \\
\hline $\begin{array}{l}\text { Froom } \\
\text { et al (51) }\end{array}$ & $P$ & $216^{d}$ & 2 & + & + & + & + & + & + & + & + & 8 & $\mathrm{HQ}$ \\
\hline $\begin{array}{l}\text { Hlatky } \\
\text { et al (31) }\end{array}$ & $P$ & $409^{b}$ & 4 & + & + & + & + & + & + & + & + & 8 & $\mathrm{HQ}$ \\
\hline $\begin{array}{l}\text { Lundbom } \\
\text { et al (27) }\end{array}$ & $P$ & $250^{b}$ & $\begin{array}{l}19-52 \\
\text { months }\end{array}$ & + & + & + & + & + & + & + & - & 7 & $\mathrm{HQ}$ \\
\hline $\begin{array}{l}\text { Mark et al } \\
(32)\end{array}$ & $P$ & $872^{d}$ & 1 & + & + & + & + & + & + & + & + & 8 & $\mathrm{HQ}$ \\
\hline $\begin{array}{l}\text { Mittag } \\
\text { et al (56) }\end{array}$ & $P$ & $132^{d}$ & 1 & + & + & + & + & + & + & + & + & 8 & $\mathrm{HQ}$ \\
\hline Munro (29) & $P$ & $79 b$ & 1.5 & + & + & + & + & + & + & + & - & 7 & $H Q$ \\
\hline $\begin{array}{l}\text { Samkange } \\
\text { et al (47) }\end{array}$ & $P$ & $751^{d}$ & 1.5 & + & + & + & + & + & + & - & + & 7 & $\mathrm{HQ}$ \\
\hline $\begin{array}{l}\text { Sellier } \\
\text { et al (33) }\end{array}$ & $P$ & $530^{b}$ & 1 & + & + & + & + & + & + & + & + & 8 & $H Q$ \\
\hline $\begin{array}{l}\text { Soderman } \\
\text { et al (48) }\end{array}$ & $P$ & $198^{d}$ & 1 & + & + & + & + & + & + & + & + & 8 & $H Q$ \\
\hline $\begin{array}{l}\text { Soejima } \\
\text { et al (45) }\end{array}$ & $P$ & $111^{c}$ & 8 months & + & + & + & + & + & - & + & + & 7 & $\mathrm{HQ}$ \\
\hline $\begin{array}{l}\text { Speziale } \\
\text { et al (52) }\end{array}$ & $P$ & $550^{b}$ & 0.5 & + & + & + & + & + & - & + & + & 7 & $H Q$ \\
\hline
\end{tabular}


quality" when meeting $\geq 7$ criteria, "medium quality" when meeting 5-6 criteria, and "low quality" when meeting $<5$ criteria. Low quality studies were excluded from the review.

\section{Data extraction}

We extracted data from the selected studies regarding study population, outcome measures, and prognostic factors. To facilitate the interpretation and comparison of the results, we categorized the studies by a specific prognostic factor. We distinguished five groups of prognostic factors for work disability based on the core concepts used in the International Classification of Functioning, Disability and Health (15): (i) disease-related factors (eg, severity of the disease, duration of the disease); (ii) body function or structural impairment factors (eg, breathlessness, pain, and body mass index); (iii) activity limitation and participation restriction factors (eg, walking, self-care, communication, and participation at work); (iv) environmental factors (eg, all factors related to work content, work environment, work conditions, care received [including medication], and situation at home); and (v) personal factors (eg, age, gender, education, coping style, and co-morbidity).

\section{Best evidence synthesis}

We synthesized the data using the "best-evidence synthesis" procedure (16). The levels of evidence were determined using a rating system similar to those used by de Croon et al (17) and Steenstra et al (18). The information was synthesized into different evidence levels as shown in figure 1. For the purpose of synthesizing the results, we clustered similar work-related outcomes - such as work disability, work disability pension, continuing to work, and return to work (not returning to work) - as the same outcome of "work disability" as there is no single or "gold standard" to define work disability. We did not cluster the outcome "sickness absence" with work disability as we believe that different prognostic factors may play a role in sickness absence (ie, "temporal" inability to work) than in work disability (ie, "permanent" inability to work). The prognostic factors predicting sickness absence were not used in the final results; the direction of the evidence level (ie, positive or negative) was based on the outcome "work disability".

Univariate or, if available, multivariate data are presented in tables. Univariate data were only shown in tables 2, 3, and 4 if there were only univariate results for the factor or if a factor was not significant in univariate analysis and, therefore, not included in the multivariate analysis.

Most articles found with the MeSH term "myocardial ischemia" dealt with a variety of coronary artery diseases including acute coronary syndrome, coronary disease, myocardial infarction, and angina pectoris. Some articles focused on return to work after a coronary artery bypass graft or a percutaneous transluminal coronary angioplasty (PTCA). In the best evidence synthesis, we considered using the categories "IHD with operation" and "IHD without operation", but we found that the diagnosis of the patients included at the

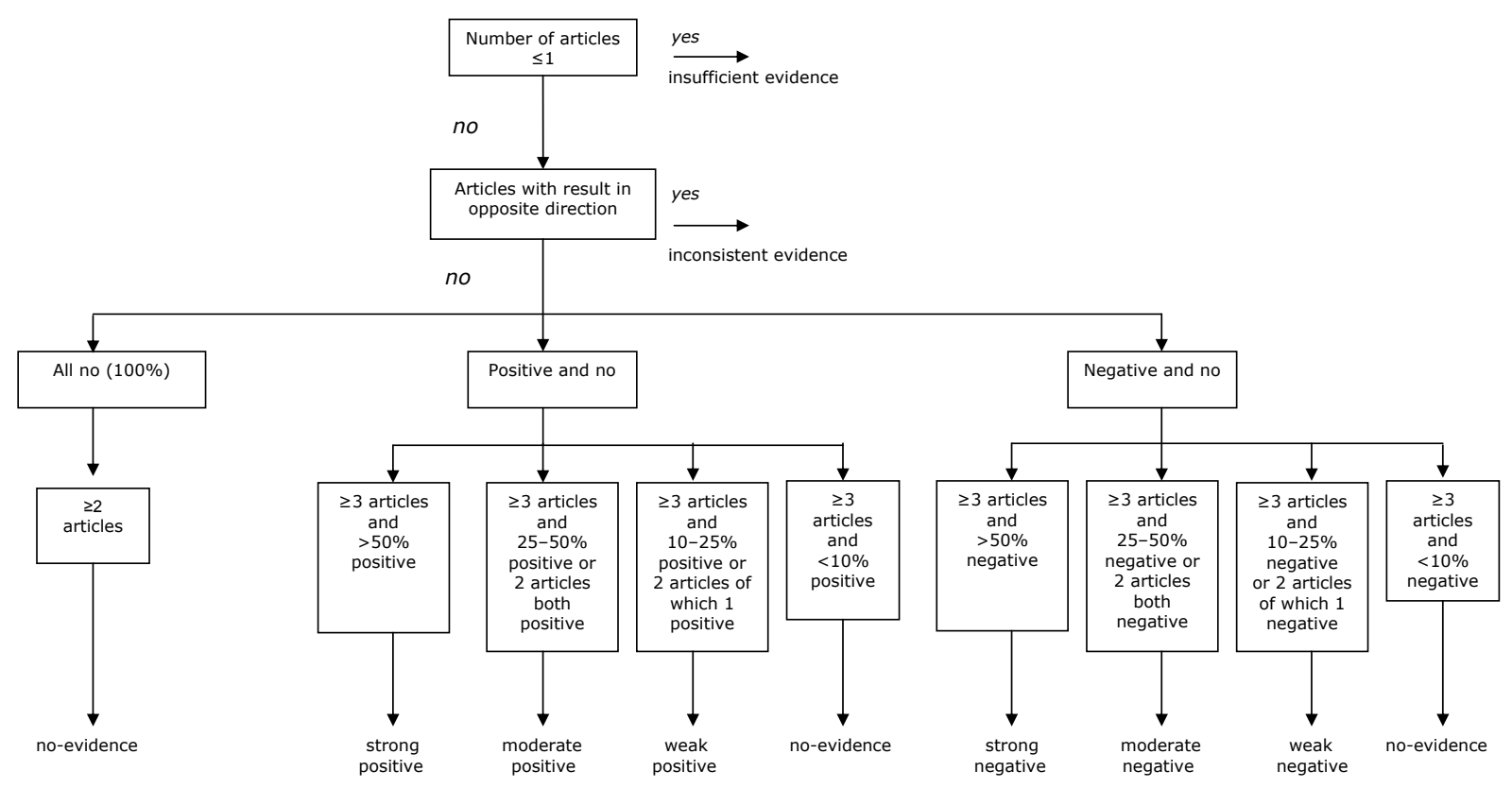

Figure 1. Best evidence synthesis 
Table 2. Results of the best evidence synthesis for employees with rheumatoid arthritis. [Positive or $(+)=$ a significant positive relation between the prognostic factor and the outcome; Negative or $(-)=\mathrm{a}$ significant negative relation between the prognostic factor and the outcome]

\begin{tabular}{|c|c|c|c|}
\hline Factor & Result & Evidence & Outcome $^{a}$ \\
\hline \multicolumn{4}{|l|}{ Disease-related factors } \\
\hline \multicolumn{2}{|l|}{ Duration of illness } & \multicolumn{2}{|l|}{ Inconsistent } \\
\hline Mau et al (35) & No effect & & Work disability \\
\hline Straaton et al (37) & No effect & & Work disability \\
\hline Reisine et al (7//22) & No effect & & Return to work \\
\hline \multicolumn{4}{|l|}{$>24$ months } \\
\hline Chung et al (25) & \multicolumn{2}{|c|}{ Positive (Nashville) b } & Work disability \\
\hline Chung et al (25) & \multicolumn{2}{|c|}{ Negative (Jyväskylä) ${ }^{\text {b }}$} & Work disability \\
\hline \multicolumn{2}{|l|}{ Disease stage } & \multicolumn{2}{|l|}{ Insufficient } \\
\hline Reisine et al (7) & No effect & & Work disability \\
\hline \multicolumn{2}{|c|}{ Non-fracture diagnosis } & Insufficient & \\
\hline Straaton et al (37) & Negative & & Return to work \\
\hline
\end{tabular}

Impairments in body function or body structure

Erythrocyte sedimentation Weak (+) rate $(30 \mathrm{~mm} / \mathrm{h})$

$\begin{array}{lll}\text { Wolfe \& Hawley (36) } & \text { No effect } & \text { Work disability } \\ \text { Young et al (26) } & \text { Positive } & \text { Work disability } \\ \text { Eberhardt \& Fex (44) } & \text { No effect } & \text { Work disability } \\ \text { Puolakka et al (24) } & \text { No effect }^{b} & \text { Work disability } \\ \text { Borg et al (43) } & \text { No effect }^{\text {b }} & \text { Work disability }\end{array}$

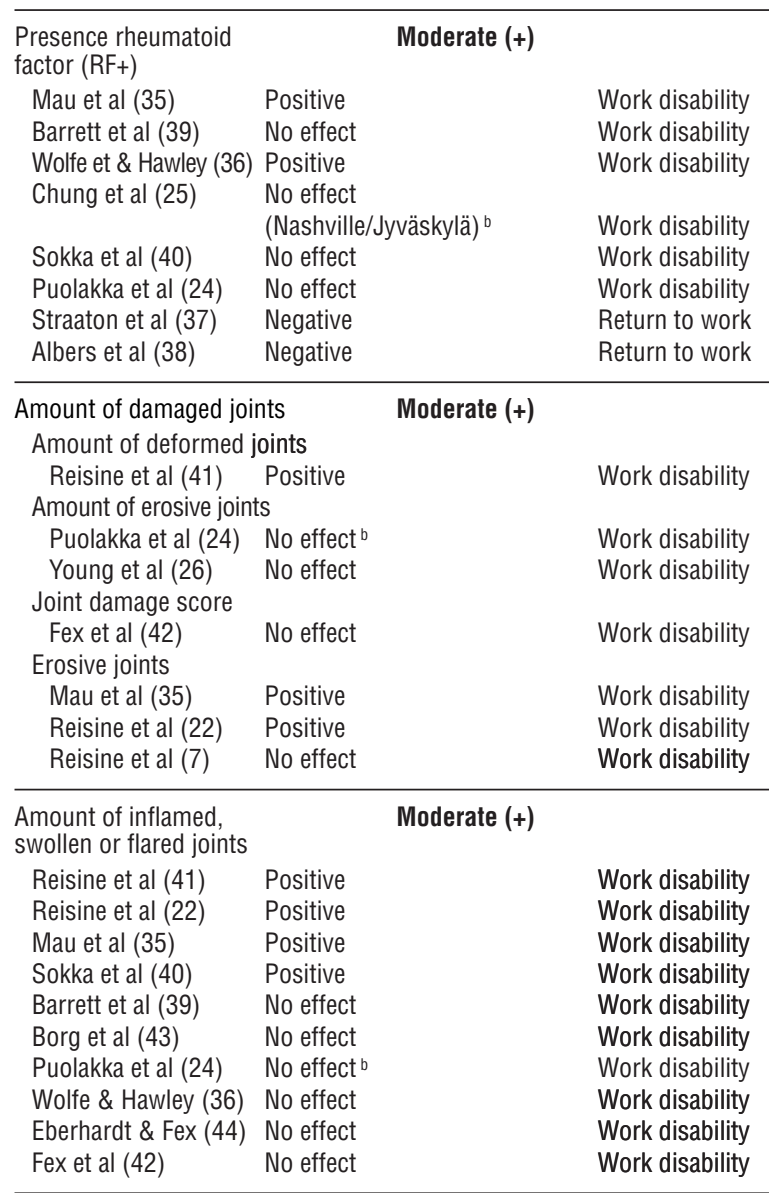

(continued)
Table 2. Continued.

\begin{tabular}{|c|c|c|c|}
\hline Factor & Result & Evidence & Outcome ${ }^{a}$ \\
\hline \multicolumn{2}{|l|}{ Amount of tender joints } & \multicolumn{2}{|l|}{ Insufficient } \\
\hline Puolakka et al (24) & No effect ${ }^{b}$ & & Work disability \\
\hline \multicolumn{2}{|l|}{ Pain } & \multicolumn{2}{|l|}{ Moderate (+) } \\
\hline \multicolumn{4}{|c|}{ Visual Analog pain scale (0-3) } \\
\hline Wolfe \& Hawley (36) & Positive & & Work disability \\
\hline \multicolumn{4}{|c|}{ Pain (high versus low score) } \\
\hline Eberhardt \& Fex (44) & No effect & & Work disability \\
\hline Reisine et al (7/22) & No effect & & Work disability \\
\hline Borg et al (43) & No effect ${ }^{b}$ & & Work disability \\
\hline \multicolumn{4}{|c|}{ Joint pain (yes versus no) } \\
\hline Puolakka et al (24) & No effect & & Work disability \\
\hline Fex et al (42) & No effect & & Work disability \\
\hline \multicolumn{4}{|c|}{ Pain interfering with work (yes versus no) } \\
\hline Mancuso et al (8) & Positive & & Work disability \\
\hline \multicolumn{4}{|c|}{ Pain at first assessment (high versus low score) } \\
\hline Chung et al (25) & $\begin{array}{l}\text { Positive } \\
\text { (Nashville/ }\end{array}$ & yväskylä) b & Work disability \\
\hline \multicolumn{4}{|c|}{ Pain at last observation } \\
\hline Chung et al (25) & No effect (I & Jashville) & Work disability \\
\hline Chung et al (25) & Positive $(J)$ & väskylä) ${ }^{b}$ & Work disability \\
\hline \multicolumn{4}{|c|}{ SF-36 (pain) (high versus low score) } \\
\hline Odergaard et al (54) & No effect & & Work disability \\
\hline \multicolumn{4}{|c|}{ Pain $>5$} \\
\hline Straaton et al (37) & Negative & & Return to work \\
\hline Keitel functional test & & Insufficient & \\
\hline Borg et al (43) & Positive ${ }^{b}$ & & Work disability \\
\hline
\end{tabular}

\begin{tabular}{|c|c|c|c|}
\hline \multirow{2}{*}{$\begin{array}{l}\text { Physician global } \\
\text { assessment (somatic) } \\
\text { Puolakka et al (24) }\end{array}$} & \multirow{2}{*}{\multicolumn{2}{|c|}{ Insufficient }} & \multirow[b]{2}{*}{ Work disability } \\
\hline & & & \\
\hline $\begin{array}{c}\text { Rheumatology Attitude } \\
\text { Odeaaard et al (18) }\end{array}$ & $\begin{array}{l}\text { Index } \\
\text { Positive }\end{array}$ & Insufficient & Wor \\
\hline
\end{tabular}

Psychological variables No evidence

90 item symptom checklist

Eberhardt et al (34) No effect Work disability

Psychological distress

Fex et al (42) No effect Work disability

Beck depression score

Borg et al (43) No effect Work disability

Arthritis Impact Insufficient

Measurement Scales affect

Odegaard et al (54) No effect Work disability

\begin{tabular}{lll}
\hline Larsen score $^{d}$ & Insufficient & \\
Jäntti et al (55) No effect & Work disability
\end{tabular}

\begin{tabular}{|c|c|}
\hline Grip strength & No evidence \\
\hline Wolfe \& Hawley (36) & No effect \\
\hline
\end{tabular}

Eberhardt \& Fex (44) No effect Work disability

Body mass index Insufficient

Wolfe \& Hawley (36) Positive Work disability

Fatigue at last observation Weak (+)

Chung et al (25) No effect (Nashville) ${ }^{b} \quad$ Work disability

\begin{tabular}{|c|c|}
\hline & Positive (Jyväskylä) b \\
\hline
\end{tabular}


Table 2. Continued.

\begin{tabular}{|c|c|c|c|}
\hline Factor & Result & Evidence & Outcome ${ }^{a}$ \\
\hline \multicolumn{4}{|c|}{ Activity limitations and participation restrictions } \\
\hline $\begin{array}{l}\text { Health Assessment } \\
\text { Questionnaire (HAQ) }\end{array}$ & \multicolumn{2}{|r|}{ Strong (+) } & \\
\hline \multicolumn{4}{|l|}{$\mathrm{HAQ}>1.50$} \\
\hline Barrett et al (39) & \multicolumn{2}{|l|}{ Positive } & Work disability \\
\hline Eberhardt \& Fex (44) & \multicolumn{2}{|l|}{ Positive } & Work disability \\
\hline Young et al (26) & \multicolumn{2}{|l|}{ Positive } & Work disability \\
\hline Fex et al (42) & \multicolumn{2}{|l|}{ Positive } & Work disability \\
\hline \multicolumn{4}{|l|}{$H A Q \geq 1.0$} \\
\hline Puolakka et al (23) & \multicolumn{2}{|l|}{ Positive } & Work disability \\
\hline Puolakka et al (24) & \multicolumn{2}{|l|}{ Positive } & Work disability \\
\hline \multicolumn{4}{|c|}{ Modified HAQ per unit } \\
\hline Odergaard et al (54) & \multicolumn{2}{|c|}{ Positive } & Work disability \\
\hline Chung et al (25) & \multicolumn{2}{|c|}{$\begin{array}{l}\text { No effect } \\
\text { (Nashville/Jyväskylä) }^{\mathrm{b}}\end{array}$} & Work disability \\
\hline \multicolumn{4}{|c|}{$\mathrm{HAQ}$ (high versus low score) } \\
\hline Wolfe \& Hawley (36) & \multicolumn{2}{|l|}{ No effect } & Work disability \\
\hline Borg et al (43) & \multicolumn{2}{|l|}{ Positive } & Work disability \\
\hline Jäntti et al (55) & \multicolumn{2}{|l|}{ Positive } & Work disability \\
\hline \multicolumn{3}{|c|}{$\begin{array}{ll}\text { American Rheumatism } & \text { Insufficient } \\
\text { Association functional class } & \end{array}$} & \\
\hline Mau et al (35) & \multicolumn{2}{|l|}{ Positive } & Work disability \\
\hline Sickness absence & & Weak (+) & \\
\hline Straaton et al (37) & No effect & & Work disability \\
\hline Reisine et al (7) & Positive & & Return to work \\
\hline
\end{tabular}

\section{Environmental factors}

\section{Occupation type}

Manual work/heavy physical

demands versus sedentary work/

professional/mental work

Eberhardt \& Fex (44)Positive

Puolakka et al (24) No effect ${ }^{b}$

Sokka et al (40) No effect

Reisine et al (41) No effect

Reisine et al (7) Positive

Manual work/heavy physical demands versus sedentary work/

professional/mental work

Young et al (26) Positive

Sokka et al (40) Positive

Wolfe \& Hawley (36) Positive

Fex et al (42) No effect

Manual versus non-manual work

Holte et al (57) Positive

Reisine et al (7) Positive

Strong (+)

\begin{tabular}{|c|c|c|}
\hline $\begin{array}{l}\text { Precision versus } \\
\text { non-precision work }\end{array}$ & & \\
\hline Borg et al (43) & Positive & Work disability \\
\hline Mau et al (35) & Positive & Work disability \\
\hline
\end{tabular}

Non-sedentary work versus Weak (+)

sedentary work

\begin{tabular}{|c|c|c|c|}
\hline $\begin{array}{l}\text { Chung et al (25) } \\
\text { Chung et al (25) }\end{array}$ & \multicolumn{2}{|c|}{$\begin{array}{l}\text { No effect (Nashville) }{ }^{b} \\
\text { Positive (Jyväskylä) }\end{array}$} & $\begin{array}{l}\text { Work disability } \\
\text { Work disability }\end{array}$ \\
\hline $\begin{array}{l}\text { Routine non-manual } \\
\text { versus professional }\end{array}$ & \multicolumn{3}{|c|}{ Insufficient } \\
\hline Holte et al (57) & Positive & & Work disability \\
\hline \multicolumn{4}{|c|}{ Autonomy at work (high versus low) No evidence } \\
\hline Eberhardt \& Fex (44) & No effect & & Work disability \\
\hline Fex et al (42) & No effect & & Work disability \\
\hline \multicolumn{2}{|l|}{ Income (high versus low) } & Insufficient & \\
\hline Holte et al (57) & No effect & & Work disability \\
\hline
\end{tabular}

Table 2. Continued.

\begin{tabular}{|c|c|c|c|}
\hline Factor & Result & Evidence & Outcome a \\
\hline \multicolumn{3}{|c|}{ Part- versus full-time work } & \\
\hline $\begin{array}{l}\text { Holte et al }(57) \\
>30 \text { hours }\end{array}$ & Positive & & Work disability \\
\hline Reisine et al (7/22) & No effect & & Work disability \\
\hline \multicolumn{2}{|c|}{ Data complexity (high versus low) } & Weak (+) & \\
\hline Wolfe \& Hawley (36) & No effect & & Work disability \\
\hline Reisine et al (22) & Positive & & Work disability \\
\hline \multicolumn{2}{|l|}{$\begin{array}{l}\text { Self-employed versus not } \\
\text { self-employed }\end{array}$} & No evidence & \\
\hline Sokka et al (40) & No effect & & Work disability \\
\hline Reisine et al (7) & No effect & & Work disability \\
\hline \multicolumn{2}{|l|}{ Manager role } & No evidence & \\
\hline \multicolumn{4}{|l|}{ Supervise others } \\
\hline Reisine et al (7) & No effect & & Work disability \\
\hline \multicolumn{4}{|c|}{ Manage people (per 1 unit) } \\
\hline Wolfe \& Hawley (36) & No effect & & Work disability \\
\hline \multicolumn{2}{|l|}{$\begin{array}{l}\text { Occupational prestige } \\
\text { (high versus low) }\end{array}$} & Insufficient & \\
\hline Reisine et al (22) & No effect & & Work disability \\
\hline \multicolumn{2}{|l|}{ Work factors } & Insufficient & \\
\hline Straaton et al (37) & No effect & & Return to work \\
\hline \multicolumn{2}{|c|}{ Duration of employment (years) } & Insufficient & \\
\hline Straaton et al (37) & No effect & & Return to work \\
\hline
\end{tabular}

Demands at work previous job Insufficient

(high versus low)

Straaton et al (37) No effect Return to work

\begin{tabular}{llll}
\hline $\begin{array}{l}\text { Prestige of previous job } \\
\text { (high versus low) } \\
\text { Straaton et al (37) }\end{array}$ & No effect & Insufficient & \\
\hline $\begin{array}{l}\text { Paid sick days }{ }^{\text {e }} \\
\text { Reisine et al (41) }\end{array}$ & Positive $^{\text {b }}$ & Insufficient & Return to work \\
\hline $\begin{array}{l}\text { Annual income (high versus low) } \\
\text { Straaton et al (37) }\end{array}$ & No effect & Insufficient & Work disability \\
\hline
\end{tabular}

Social security insurance Insufficient

(yes versus no)

Straaton et al (37) Negative Return to work

\begin{tabular}{ll}
\hline Type of drug therapy & No evidence \\
Combination treatment & \\
Puolakka et al (24) No effect ${ }^{\text {a }}$ & \\
Treatment group (disease- & Work disability \\
modifying anti-rheumatic drug) & \\
Borg et al (43) No effect & Work disability \\
Eberhardt \& Fex (44) No effect & Work disability \\
\hline
\end{tabular}

\begin{tabular}{|c|c|c|}
\hline \multicolumn{3}{|c|}{ Ever/never biological use Insufficient } \\
\hline Chung et al (25) & $\begin{array}{l}\text { No effect } \\
\text { (Nashville/Jyväskylä) b }\end{array}$ & Work disability \\
\hline Ever/never methotrexate & e $\quad$ Weak (+) & \\
\hline Chung et al (25) & Positive (Jyväskylä) ${ }^{\mathrm{b}}$ & Work disability \\
\hline Chung et al (25) & No effect (Nashville) ${ }^{b}$ & Work disability \\
\hline
\end{tabular}

Ever/never used prednisone Weak (+)

$\begin{array}{ccc}\text { Chung et al (25) } & \text { Positive (Jyväskylä) } & \\ & & \text { Work disability }\end{array}$

Chung et al (25) No effect (Nashville) ${ }^{\text {b }} \quad$ Work disability 
Table 2. Continued.

\begin{tabular}{|c|c|c|c|}
\hline Factor & Result & Evidence & Outcome a \\
\hline \multicolumn{2}{|c|}{ Time before start of drug therapy } & Weak (-) & \\
\hline \multicolumn{4}{|c|}{ Delay to treatment (>4 months) } \\
\hline Puolakka et al (24) & \multicolumn{2}{|l|}{ No effect ${ }^{a}$} & Work disability \\
\hline \multicolumn{4}{|c|}{ Time to first disease-modifying anti-rheumatic drug } \\
\hline Chung et al (25) & \multicolumn{2}{|c|}{ Negative (Nashville) b } & Work disability \\
\hline Chung et al (25) & \multicolumn{2}{|c|}{ No effect (Jyväskylä) b } & Work disability \\
\hline
\end{tabular}

\section{Personal factors}

Age

High versus low

Odergaard et al (54) No effect

Eberhardt \& Fex (44) No effect

Puolakka et al (23/24) Positive

Reisine et al (7/22) Positive

Borg et al (43) Positive

Mau et al (35) Positive

Higher age

Albers et al (38) Positive

Per 5 years extra of age

Sokka et al (40) Positive

$40-49$ versus $30-39 / 50-56$ versus 39 years

Holte et al (57) Positive

$\leq 55$ versus $>55$ years

Puolakka et al (23/24) Positive

$46-55$ versus $\geq 35 />55$ versus $<35$ years

Fex et al (42) Positive

$46-55$ versus $\leq 35 />55$ versus $<35$ years

Chung et al (25) Positive (Jyväskylä) b

Chung et al (25) No effect (Nashville) ${ }^{b}$

$>43$ versus $\leq 43$ years

Straaton et al (37) Negative

Work disability

Work disability

Work disability

Work disability

Work disability

Work disability

Work disability

Work disability

Work disability

Work disability

Work disability

Work disability

Work disability

Return to work

Age at onset Weak $(+$

Young et al (26) Positive

$<50$ years versus $>50$ years

Barrett et al (24) No effect

Work disability

Gender (female versus male)

Reisine et al (7/22) No effect

Odergaard et al (54) Positive

Holte et al (57) Positive

Barrett et al (24) No effect

Albers et al (38) Positive

Fex et al (42) No effect

Wolfe \& Hawley (36) Positive

Young (26) Positive

Borg et al (43) No effect ${ }^{b}$

Eberhardt \& Fex (44) No effect

Puolakka et al (24) No effect ${ }^{b}$

Sokka et al (40) No effect

Chung et al (25) No effect ${ }^{b}$

Straaton et al (37) No effect

\begin{tabular}{|c|c|c|}
\hline \multicolumn{3}{|c|}{ No evidence } \\
\hline \multicolumn{3}{|c|}{ Married versus single/divorced/ widowed } \\
\hline Reisine et al (7/22) & No effect & Work disability \\
\hline Eberhardt \& Fex (44) & No effect & Work disability \\
\hline Albers et al (38) & No effect & Work disability \\
\hline Borg et al (43) & No effect & Work disability \\
\hline Fex et al (42) & No effect & Work disability \\
\hline Sokka et al (40) & No effect & Work disability \\
\hline Holte et al (57) & No effect & Work disability \\
\hline \multicolumn{3}{|c|}{ Married (only in women) } \\
\hline Straaton et al (37) & No effect & Return to work \\
\hline Reisine et al (41) & Positive & Work disability \\
\hline
\end{tabular}

(continued)
Table 2. Continued

\begin{tabular}{|c|c|c|c|}
\hline Factor & Result & Evidence & Outcome ${ }^{a}$ \\
\hline
\end{tabular}

Holte et al (57) Positive Work disability

Desire to be or remain employed Weak (-)

(higher versus low)

Reisine et al (7) No effect Work disability

Reisine et al (22) Negative Work disability

Satisfaction with working

conditions (high versus low)

Eberhardt \& Fex (44) No effect

Fex et al (42) No effect

Reisine et al (41) Negative

Weak (-)

Education

Work disability

Work disability

Work disability

High versus low

Reisine et al (7/22) No effect

Sokka et al (40) No effect

Odegard (54) Negative

Borg et al (43) No effect ${ }^{b}$

Fex et al (42) Positive

Straaton et al (37) Positive

Higher

Wolfe \& Hawley (36) Negative

Eberhardt \& Fex (44) No effect

Puolakka et al (24) No effect

Number of years

Barrett et al (39) No effect

Moderate (-)

0-9 years

Holte et al (57) Negative

$>12$ years

Chung et al (25) Negative

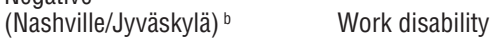

\begin{tabular}{llll}
\hline $\begin{array}{l}\text { Social class } \\
\text { Barrett et al (39) }\end{array}$ No effect & Insufficient & Work disability \\
\hline
\end{tabular}

Ethnic origin (white Moderate (-)

versus non-white)

Wolfe \& Hawley (36) No effect

Negative (Nashville) b ${ }^{b} \quad$ Work disability

Reisine et al (41) No effect Work disability

\begin{tabular}{ccc}
\hline Co-morbidity (yes versus no) & No evidence & \\
Odergaard et al (54) & No effect & \\
Straaton et al (37) & No effect & Work disability \\
\hline
\end{tabular}

\begin{tabular}{lll}
\hline $\begin{array}{l}\text { General health } \\
\text { Borg et al (43) }\end{array}$ Insufficient & \\
No effect ${ }^{\mathrm{b}}$ & & Work disability
\end{tabular}

Patient Global Severity Score Moderate (+)

High versus low

Wolfe \& Hawley (36) No effect Work disability

Chung et al (25) Positive

(Nashville/Jyväskylä) ${ }^{b} \quad$ Work disability

Puolakka et al (24) Positive

Work disability

At first assessment

(high versus low)

Chung et al (25) No effect (Nashville) ${ }^{\text {b }} \quad$ Work disability

At last assessment

(high versus low)

Chung et al (25) Positive (Jyväskylä) ${ }^{\text {b }} \quad$ Work disability

a Based on the best evidence synthesis level

${ }^{b}$ Results based on univariate analysis.

c Helplessness subscale (RAl-score $<$ =median).

d Rating scale mainly for hip impairments.

e $<1$ week versus $\geq 1$ week. 
Table 3. Results of the best evidence synthesis for employees with asthma. [Positive or $(+)=$ a significant positive relation between the prognostic factor and the outcome; Negative or $(-)=\mathrm{a}$ significant negative relation between the prognostic factor and the outcome; Cont. employ = continuous employment]

\begin{tabular}{|c|c|c|c|}
\hline Factor & Result & Evidence & Outcome a \\
\hline \multicolumn{4}{|l|}{ Disease-related factors } \\
\hline Asthma severity score & & Weak (+) & \\
\hline Blanc et al (10) & Positive & & Work disability \\
\hline Yelin et al (11) & No effect & & Cont. employ \\
\hline
\end{tabular}

\begin{tabular}{|c|c|c|c|}
\hline \multicolumn{4}{|c|}{ Impairments in body function or body structure } \\
\hline \multicolumn{2}{|c|}{ Forced Expiratory Volume (\%) } & \multirow{2}{*}{ Insufficient } & \multirow[b]{2}{*}{ Work disability } \\
\hline Blanc et al (10) & Positive & & \\
\hline \multicolumn{4}{|c|}{ Activity limitations and participation restrictions } \\
\hline SF-36 (physical) & & Insufficient & \\
\hline Yelin et al (11) & No effec & & Cont. employ \\
\hline
\end{tabular}

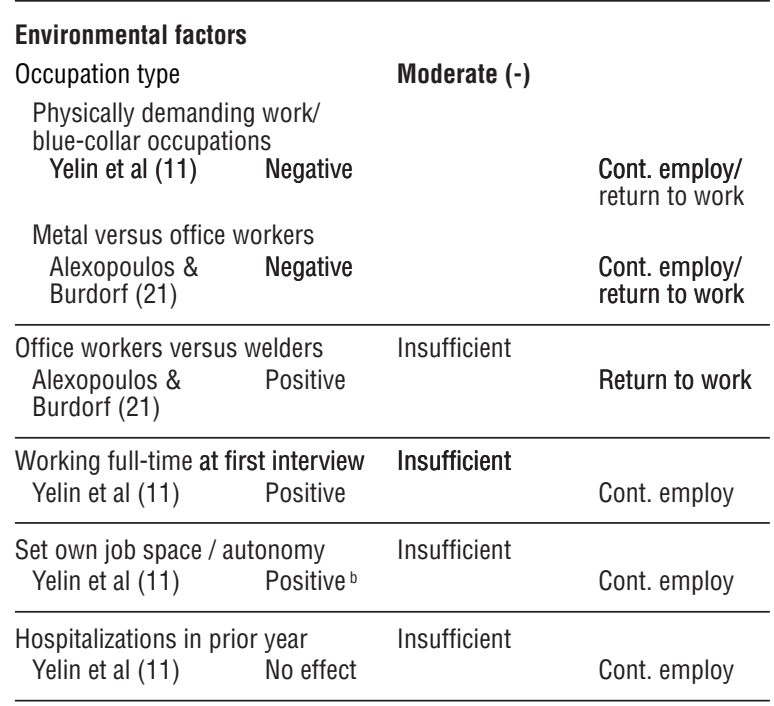

\section{Personal factors}

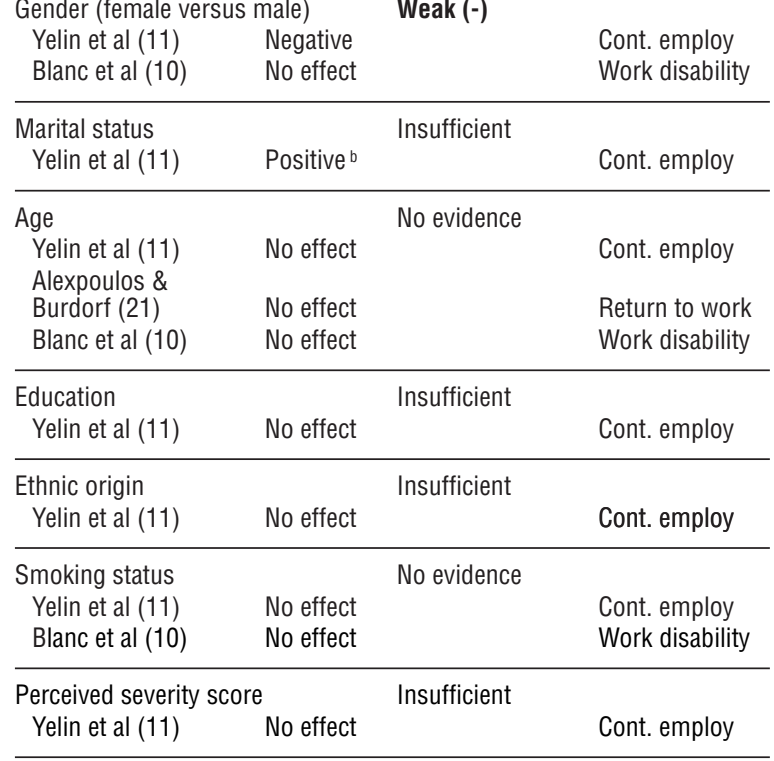

${ }^{a}$ Based on the best evidence synthesis level

${ }^{\mathrm{b}}$ Results based on univariate analysis.
Table 4. Results of the best evidence synthesis for employees with ischemic heart disease. [Positive or $(+)=$ a significant positive relation between the prognostic factor and the outcome; Negative or $(-)=$ a significant negative relation between the prognostic factor and the outcome; $\mathrm{CBS}=$ coronary bypass surgery]

\begin{tabular}{lll}
\hline Factor & Result & Evidence \\
\hline
\end{tabular}

\section{Disease-related factors}

History of myocardial infarction No evidence (yes versus no)

$\begin{array}{lll}\text { Lundbom et al (27) } & \text { No effect }{ }^{\mathrm{b}} & \text { Return to work } \\ \text { Sellier et al (33) } & \text { No effect } & \text { Return to work }\end{array}$

\section{Presence of congestive heart failure Weak (-)}

\begin{tabular}{|c|c|c|c|}
\hline $\begin{array}{l}\text { Hlatky et al (31) } \\
\text { Mark et al (32) }\end{array}$ & $\begin{array}{l}\text { No effect } \\
\text { Negative }\end{array}$ & & $\begin{array}{l}\text { Return to work } \\
\text { Return to work }\end{array}$ \\
\hline \multicolumn{2}{|c|}{ Extent of coronary artery disease } & \multirow[t]{2}{*}{ Insufficient } & \\
\hline Hlatky et al (31) & No effect & & Return to work \\
\hline
\end{tabular}

\begin{tabular}{|c|c|c|c|}
\hline \multicolumn{2}{|c|}{$\begin{array}{l}\text { Diagnosed with myocardial } \\
\text { infarction (versus angina) }\end{array}$} & \multirow[t]{2}{*}{ Insufficient } & \multirow[b]{2}{*}{ Return to work } \\
\hline Earle et al (58) & Positive & & \\
\hline $\begin{array}{l}\text { Chronic heart faill } \\
\text { Mark et al (32) }\end{array}$ & I) & Insufficient & to mol \\
\hline
\end{tabular}

Evidence of extracardiag vascular

disease (yes versus no) Insufficient

Mark et al (32) Negative Return to work

\begin{tabular}{lrr}
\hline $\begin{array}{l}\text { Presence of angina pectoris } \\
\text { (yes versus no) }\end{array}$ & Weak (-) & \\
Sellier et al (33) $\quad$ Negative & & Return to work
\end{tabular}

$\begin{array}{lll}\text { Sellier et al (33) } & \text { Negative } & \text { Return to work } \\ \text { Mark et al (32) } & \text { No effect } & \text { Return to work }\end{array}$

Presence of angina before acute Insufficient myocardial infarction

Froom et al (51) Negative Return to work

Angina course (stable or none Insufficient versus progressive angina versus unstable angina)

\begin{tabular}{|c|c|c|c|}
\hline Mark et al (32) & No effect & & Return to work \\
\hline Duration of angina & & Insufficient & \\
\hline Lundbom et al (27) & No effect ${ }^{b}$ & & $\begin{array}{l}\text { Working full- } \\
\text { time after CBS }\end{array}$ \\
\hline
\end{tabular}

\begin{tabular}{|c|c|c|c|}
\hline $\begin{array}{l}\text { History of Percutar } \\
\text { luminal Coronary } A\end{array}$ & $\begin{array}{l}\text { Trans- } \\
\text { lasty }\end{array}$ & Weak (+) & \\
\hline Sellier et al (33) & No effect & & Return to work \\
\hline Drory et al (34) & Positive & & Return to work \\
\hline
\end{tabular}

History of coronary artery Insufficient

bypass graft

Sellier et al (33) No effect Return to work

Acute versus elective operation Insufficient Return to work

Lundbom et al (27) Positive ${ }^{b}$

Complete revascularization ${ }^{c} \quad$ Weak (+)

Sellier et al (33) No effect $\quad$ Return to work
Boudrez \&

De Backer ${ }^{d}(19) \quad$ Positive Return to work

Number of coronary vessels involved No evidence

Sellier et al (33) No effect $\quad$ Return to work

Mark et al (32) No effect Return to work

(continued) 
Table 4. Continued.

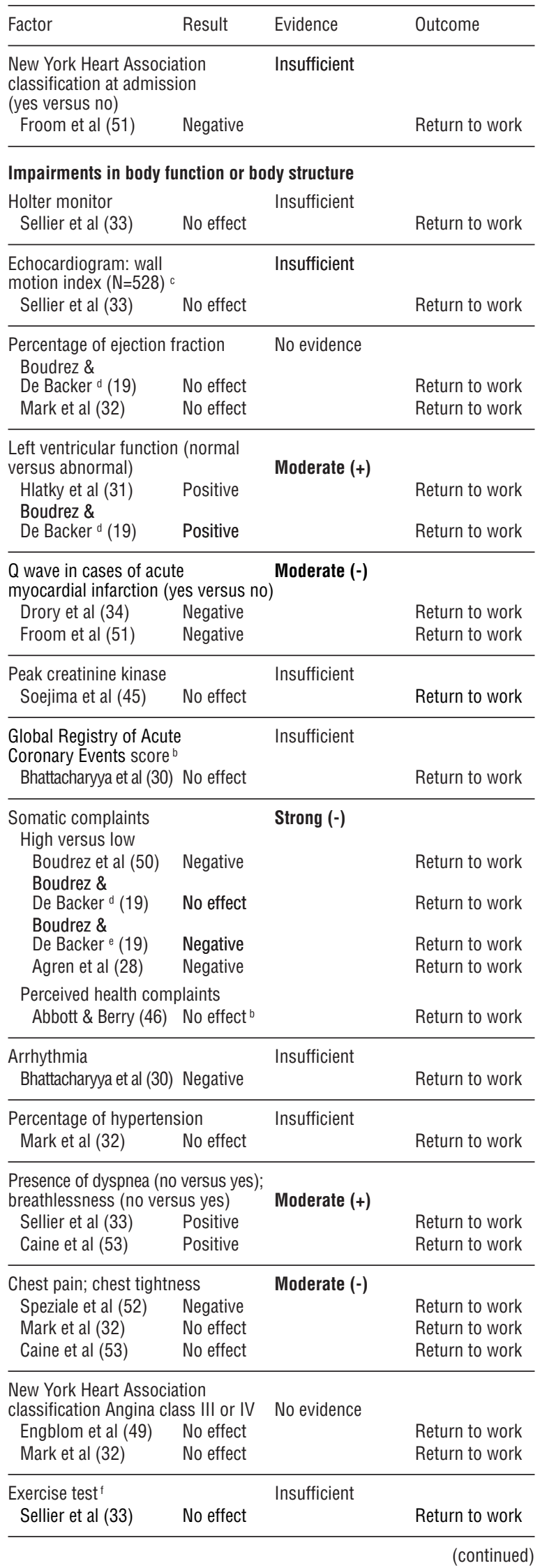

Table 4. Continued.

\begin{tabular}{|c|c|c|c|}
\hline Factor & Result & Evidence & Outcome \\
\hline $\begin{array}{l}\text { Peak workflow (watts); } \\
\text { final workflow }\end{array}$ & & Weak (+) & \\
\hline Sellier et al (33) & Positive & & Return to work \\
\hline Engblom et al (49) & No effect & & Return to work \\
\hline
\end{tabular}

\begin{tabular}{|c|c|c|c|}
\hline \multirow{2}{*}{$\begin{array}{l}\text { Exercise duration } \\
<420 \text { seconds } \\
\text { Sellier et al (33) }\end{array}$} & \multicolumn{3}{|c|}{ Insufficient } \\
\hline & Negative & & Return to work \\
\hline Exercise tolerance & & No evidence & \\
\hline Boudrez et al (50) & No effect & & Return to work \\
\hline Boudrez \& & & & \\
\hline De Backer e (19) & No effect & & Return to work \\
\hline Söderman et al (48) & No effect & & Return to work \\
\hline $\begin{array}{l}\text { Anxiety (clinical/border } \\
\text { versus normal) }\end{array}$ & & No evidence & \\
\hline Samkange et al (47) & No effect & & Return to work \\
\hline De Backer ${ }^{\mathrm{e}}(19)$ & No effect & & Return to work \\
\hline Abbott \& Berry (46) & No effect & & Return to work \\
\hline
\end{tabular}

Depression \& anxiety

Strong (-)

Beck depression score

Bhattacharyya et al (30) Negative

Söderman et al (48) Negative

Beck depression score (preoperative)

Engblom et al (49) No effect

Depressive symptoms in hospital

Soejima et al (45) Negative

Return to work

Depression (clinical/

borderline versus normal)

Abbott \& Berry (46) Negative ${ }^{b}$

Return to work

Return to work

Hospital anxiety and depression scale

adjusted for Germany

Samkange et al (47) Negative Return to work

\begin{tabular}{ccc}
\hline $\begin{array}{c}\text { Mental health inventory score } \\
\text { Hlatky et al (31) } \quad \text { No effect }\end{array}$ & Insufficient & Return to work
\end{tabular}

Summary psychological scales - Weak $(+)$

Weak (+)

Boudrez \&

De Backer e (19) No effect Return to work

Mark et al (32) Positive Return to work

Cognitive insufficiency No evidence

Boudrez \&

De Backer ${ }^{\mathrm{e}}(19) \quad$ No effect Return to work

Boudrez \&

De Backer ${ }^{d}(19) \quad$ No effect Return to work

Psycho neuroticism Insufficient

Boudrez \&
De Backer ${ }^{d}$ (19) No effect $\quad$ Return to work

\begin{tabular}{lll}
\hline Extraversion & Insufficient & \\
Soejima et al (45) & Positive & Return to work \\
\hline
\end{tabular}

\section{Activity limitations and participation restrictions}

Duke activity status index Weak (-)

Functional activity patients view

Hlatky et al (31) No effect Return to work

Duke activity (0-12)

Mark et al (32) Negative Return to work 
Table 4. Continued.

\begin{tabular}{|c|c|c|c|}
\hline Factor & Result & Evidence & Outcome \\
\hline Subjective working capa & acity & Strong (-) & \\
\hline \multicolumn{4}{|c|}{$\begin{array}{l}\text { Vocational disability to cardiac status } \\
\text { (patient's view) (strong versus light) }\end{array}$} \\
\hline Mittag et al (56) & Negative & & $\begin{array}{l}\text { Working full- } \\
\text { time after CBS }\end{array}$ \\
\hline \multicolumn{4}{|c|}{$\begin{array}{l}\text { Patients' perception of their working } \\
\text { capacity } 6 \text { months after CBS }\end{array}$} \\
\hline Engblom et al (49) & No effect & & Return to work \\
\hline \multicolumn{4}{|c|}{$\begin{array}{l}\text { Work incapacitation (strong versus } \\
\text { moderate versus light) }\end{array}$} \\
\hline Samkange et al (47) & Negative & & Return to work \\
\hline \multicolumn{2}{|c|}{$\begin{array}{l}\text { Vocational disability due to } \\
\text { overall health (physician's view) }\end{array}$} & Insufficient & \\
\hline Mittag et al (56) & Positive & & Return to work \\
\hline \multicolumn{2}{|c|}{ Sick listed before operation } & Strong (-) & \\
\hline Lundbom et al (27) & Negative ${ }^{b}$ & & $\begin{array}{l}\text { Working full- } \\
\text { time after CBS }\end{array}$ \\
\hline Munro (29) & Negative & & Return to work \\
\hline Agren et al (28) & Negative & & Return to work \\
\hline Engblom et al (49) & No effect & & Return to work \\
\hline Caine et al (53) & Negative & & Return to work \\
\hline $\begin{array}{l}\text { Boudrez \& } \\
\text { De Backer }{ }^{d}(19)\end{array}$ & Negative & & Return to work \\
\hline \multicolumn{2}{|c|}{ Work status before surgery } & Insufficient & \\
\hline Speziale et al (52) & No effect & & Return to work \\
\hline \multicolumn{2}{|c|}{ Sick-leave versus laid-off } & Insufficient & Return to work \\
\hline \multicolumn{2}{|c|}{ Effect of health on activities } & Insufficient & \\
\hline Mark et al (32) & No effect & & Return to work \\
\hline \multicolumn{2}{|c|}{$\begin{array}{l}\text { Nottingham health profile } \\
\text { (physical mobility, lower } \\
\text { than twice normal population) }\end{array}$} & Insufficient & \\
\hline Caine et al (53) & Negative & & Return to work \\
\hline \multicolumn{4}{|l|}{ Environmental factors } \\
\hline \multicolumn{2}{|c|}{$\begin{array}{l}\text { Occupation type } \\
\text { Blue- versus white-collar }\end{array}$} & Moderate (-) & \\
\hline \multicolumn{3}{|c|}{$\begin{array}{l}\text { Blue- versus white-collar } \\
\text { Boudrez et al (50) No effect }\end{array}$} & $\begin{array}{l}\text { Working full- } \\
\text { time after CBS }\end{array}$ \\
\hline Hlatky et al (31) & \multicolumn{2}{|l|}{ No effect } & $\begin{array}{l}\text { Return to work } \\
\text { after CBS }\end{array}$ \\
\hline Caine et al (53) & \multicolumn{2}{|l|}{ No effect } & $\begin{array}{l}\text { Return to work } \\
\text { after CBS }\end{array}$ \\
\hline Drory et al (34) & \multicolumn{2}{|l|}{ Negative } & Return to work \\
\hline \multicolumn{3}{|c|}{ Samkange et al (47) Negative } & Return to work \\
\hline \\
\hline \multicolumn{3}{|c|}{$\begin{array}{l}\text { Goods-producing industry } \\
\text { Engblom et al (49) No effect }\end{array}$} & $\begin{array}{l}\text { Working full- } \\
\text { time after CBS }\end{array}$ \\
\hline \multicolumn{4}{|c|}{ Manual/white-collar work } \\
\hline \multicolumn{3}{|c|}{ Lundboom et al (27) No effect ${ }^{b}$} & $\begin{array}{l}\text { Working full- } \\
\text { time after CBS }\end{array}$ \\
\hline $\begin{array}{l}\text { Boudrez \& } \\
\text { De Backer }{ }^{\mathrm{e}}(19)\end{array}$ & \multicolumn{2}{|l|}{ Negative } & $\begin{array}{l}\text { Working full- } \\
\text { time after CBS }\end{array}$ \\
\hline \multicolumn{4}{|c|}{ Physically demanding work } \\
\hline Soejima et al (45) & No effect & & $\begin{array}{l}\text { Return to work } \\
\text { after CBS }\end{array}$ \\
\hline Sellier et al (33) & Negative & & $\begin{array}{l}\text { Return to work } \\
\text { after CBS }\end{array}$ \\
\hline
\end{tabular}

Table 4. Continued.

\begin{tabular}{|c|c|c|c|}
\hline Factor & Result & Evidence & Outcome \\
\hline \multicolumn{2}{|c|}{$\begin{array}{l}\text { Self-employed tradesmen versus } \\
\text { public service employees }\end{array}$} & \multirow[t]{2}{*}{ Insufficient } & \\
\hline Lundbom et al (27) & No effect ${ }^{b}$ & & $\begin{array}{l}\text { Working full- } \\
\text { time after CBS }\end{array}$ \\
\hline Full- versus part-time & & Insufficient & \\
\hline Hlatky et al (31) & Positive & & Return to work \\
\hline \multicolumn{2}{|l|}{ Hours worked per week } & Insufficient & \\
\hline Mark et al (32) & No effect & & Return to work \\
\hline \multicolumn{2}{|c|}{ Length of time with an employer } & Insufficient & \\
\hline Caine et al (53) & No effect ${ }^{b}$ & & Return to work \\
\hline Mental stress of job & & No evidence & \\
\hline Hlatky et al (31) & No effect & & Return to work \\
\hline Mark et al (32) & No effect & & Return to work \\
\hline Flexible work schedule & & Insufficient & \\
\hline Earle et al (58) & No effect & & Return to work \\
\hline \multicolumn{2}{|c|}{ Able to work fewer hours } & Insufficient & \\
\hline Earle et al (58) & No effect & & Return to work \\
\hline \multicolumn{2}{|c|}{ Allowed to change work tasks } & Insufficient & \\
\hline Earle et al (58) & No effect & & Return to work \\
\hline \multicolumn{2}{|c|}{ Job held open after operation } & Insufficient & \\
\hline Caine et al (53) & No effect & & Return to work \\
\hline \multicolumn{2}{|l|}{$\begin{array}{l}\text { Decision latitude/authority } \\
\text { Boudrez \& }\end{array}$} & Insufficient & \\
\hline $\begin{array}{l}\text { Boudrez \& } \\
\text { De Backer }{ }^{d}(19)\end{array}$ & No effect & & Return to work \\
\hline \multicolumn{2}{|c|}{$\begin{array}{l}\text { Psychological job demands } \\
\text { Boudrez \& }\end{array}$} & Insufficient & \\
\hline $\begin{array}{l}\text { Boudrez \& } \\
\text { De Backer }{ }^{d}(19)\end{array}$ & No effect & & Return to work \\
\hline \multirow{2}{*}{$\begin{array}{l}\text { Method of pay } \\
\text { Hlatky et al (31) }\end{array}$} & & Insufficient & \\
\hline & No effect & & Return to work \\
\hline \multicolumn{2}{|l|}{ Income } & No evidence & \\
\hline $\begin{array}{l}\text { Annual income job; } \\
\text { Hlatky et al (31) }\end{array}$ & No effect & & Return to work \\
\hline Percentage of income & No effect & & Return to work \\
\hline \multicolumn{2}{|c|}{ Job insecurity (high versus low) } & Insufficient & \\
\hline $\begin{array}{l}\text { Boudrez \& } \\
\text { De Backer }{ }^{d}(19)\end{array}$ & Positive & & Return to work \\
\hline \multicolumn{2}{|c|}{ Paid leave available at work } & Insufficient & \\
\hline Earle et al (58) & Positive & & Return to work \\
\hline \multicolumn{2}{|c|}{ Co-workers/supervisor support } & Insufficient & \\
\hline Earle et al (58) & No effect & & Return to work \\
\hline \multicolumn{2}{|c|}{ Social support from friends } & Weak (+) & \\
\hline Boudrez et al (50) & Positive & & Return to work \\
\hline Soejima et al (45) & No effect & & Return to work \\
\hline $\begin{array}{l}\text { Boudrez \& } \\
\text { De Backer }{ }^{d}(19)\end{array}$ & No effect & & Return to work \\
\hline \multirow{3}{*}{$\begin{array}{l}\text { Health insurance } \\
\text { Hlatky et al (31) } \\
\text { Earle et al (58) }\end{array}$} & & Weak (+) & \\
\hline & Positive & & Return to work \\
\hline & No effect & & Return to work \\
\hline \multicolumn{2}{|l|}{$\begin{array}{l}\text { Other household members } \\
\text { with job }\end{array}$} & Insufficient & \\
\hline Hlatky et al (31) & No effect & & Return to work \\
\hline
\end{tabular}


Table 4. Continued.

\begin{tabular}{|c|c|c|c|}
\hline Factor & Result & Evidence & Outcome \\
\hline \multicolumn{2}{|c|}{ Employment status partner } & \multirow[t]{2}{*}{ Insufficient } & \\
\hline Mark et al (32) & No effect & & Return to work \\
\hline \multicolumn{2}{|c|}{ Husband has higher education } & \multirow[t]{2}{*}{ Insufficient } & \\
\hline Earle et al (58) & No effect & & Return to work \\
\hline \multirow{2}{*}{\multicolumn{2}{|c|}{$\begin{array}{l}\text { Number of dependents } \\
\text { Mark et al (32) No effect }\end{array}$}} & \multirow{2}{*}{ Insufficient } & \\
\hline & No effect & & Return to work \\
\hline \multirow{2}{*}{\multicolumn{2}{|c|}{$\begin{array}{l}\text { Residence } \\
\text { Rural versus urban area } \\
\text { Lundbom et al (27) No effect }{ }^{b}\end{array}$}} & \multirow[t]{6}{*}{ Moderate (-) } & \\
\hline & & & $\begin{array}{l}\text { Working full- } \\
\text { time after CBS }\end{array}$ \\
\hline \multirow{4}{*}{\multicolumn{2}{|c|}{$\begin{array}{l}\text { South East region France } \\
\text { Sellier et al (33) Negative } \\
\text { Region of residence (south Italy) } \\
\text { Speziale et al (52) No effect }\end{array}$}} & & \\
\hline & & & Return to work \\
\hline & & & \\
\hline & & & Return to work \\
\hline \multicolumn{2}{|c|}{$\begin{array}{l}\text { Length of time waiting for } \\
\text { grafting ( }<6 \text { months })\end{array}$} & Insufficient & \\
\hline Caine et al (53) & Positive & & Return to work \\
\hline \multicolumn{2}{|c|}{ Attended cardiac rehabilitation } & Moderate (+) & \\
\hline Earle et al (58) & Positive & & Return to work \\
\hline Boudrez et al (50) & Positive & & Return to work \\
\hline \multicolumn{2}{|c|}{ Anti-depressant use } & Insufficient & \\
\hline Bhattacharyya et al (30) & No effect & & Return to work \\
\hline \multicolumn{2}{|c|}{$\begin{array}{l}\text { Time interval between acute } \\
\text { myocardial infarction \& visit } \\
\text { to occupational medicine clinic }\end{array}$} & Insufficient & \\
\hline Froom et al (51) & Negative & & Return to work \\
\hline
\end{tabular}

\section{Personal factors}

Age

High versus low

Agren et al (28) Negative

Söderman et al (48) Negative Lundbom et al (27) Negative Caine et al (53) No effect Boudrez et al (50) No effect Soejima et al (45) No effect Abbott \& Berry (46) Negative $>51$ years

Sellier et al (33) Negative

Speziale et al (52) Negative

$>55$ years

Drory et al (34) Negative

$>54$ years

Froom et al (51) Negative

Mark et al (32) Negative

$52-56 / 57-61$ versus $<52$ years

Samkange et al (47) Negative

$<55$ versus $>55$ years

Söderman et al (48) Negative

$46-55 / 56-53$ versus $<45$ years

Munro (29) Negative

Per extra year

Mittag et al (56) Negative

$<55$ years versus $55-59$ years

Hlatky et al (31) Negative

In years

Bhattacharyya

et al (30) Negative

\section{Strong (-)}

Working fulltime after CBS Return to work Return to work Return to work Return to work Return to work Return to work

Return to work Return to work

Return to work

Return to work

Return to work

Return to work

Return to work

Return to work

Return to work

Return to work

Return to work

(continued)
Table 4. Continued.

\begin{tabular}{ll}
\hline Factor & Result \\
\hline Gender (male versus female) & No effect \\
Sellier (28) & Positive \\
Hlatky et al (31) & No effect \\
Bhattacharyya et al (30) & No effect \\
Drory et al (34) & No effect \\
Samkange et al (47) & No effect \\
Mark et al (32) & No effect
\end{tabular}

Education

Strong (+)

High versus low

Earle et al (58) Positive

Soejima et al (45) No effect

Söderman et al (48) Positive

$\geq 15$ versus $9-11$ versus $\leq 8$ years

Mark et al (32) Positive

Evidence

Outcome

Weak (+)

Return to work

Return to work.

Return to work

Return to work

Return to work

Return to work

\begin{tabular}{|c|c|c|c|}
\hline $\begin{array}{l}\text { Professional education } \\
\text { Engblom et al (49) }\end{array}$ & No effect & Insufficient & Return to work \\
\hline $\begin{array}{l}\text { Socioeconomic status } \\
\text { Engblom et al (49) }\end{array}$ & No effect & Weak (-) & Return to work \\
\hline $\begin{array}{l}\text { Social class (high vers } \\
\text { Abbott \& Berry (46) }\end{array}$ & $\begin{array}{l}\text { sus low) } \\
\text { Negative }\end{array}$ & & Return to work \\
\hline
\end{tabular}

\begin{tabular}{|c|c|c|c|}
\hline Marital status & & No evidence & \\
\hline Earle et al (58) & No effect & & Return to work \\
\hline Mark et al (32) & No effect & & Return to work \\
\hline
\end{tabular}

Origin (Israel/ Europe/ Insufficient

America versus other)

Drory et al (34) Positive Return to work

Ethnic origin Weak (+)

White versus non-white

Earle et al(32) No effect

Return to work

Return to work

Return to work

White versus black

Mark et al (32) Positive

Return to work

\begin{tabular}{|c|c|c|}
\hline IVlark et al (32) & & Return to work \\
\hline \multicolumn{3}{|c|}{ Smoking No evidence } \\
\hline \multicolumn{3}{|c|}{ Smoker versus non-smoker } \\
\hline Earle et al (58) No effect & & Return to work \\
\hline \multicolumn{3}{|l|}{ Smoking history (percentage) } \\
\hline Mark et al (32) $\quad$ No effect & & Return to work \\
\hline Active versus passive coping & Insufficient & \\
\hline Agren et al (28) Positive & & $\begin{array}{l}\text { Working full- } \\
\text { time after CBS }\end{array}$ \\
\hline
\end{tabular}

Health locus of control (internal Moderate (+)

versus external)

Soejima et al (45) No effect

Earle et al (58) No effect

Return to work

Abbott \& Berry (46) Positive ${ }^{a}$

Return to work

Boudrez \&

Return to work

De Backer ${ }^{d}(19) \quad$ No effect

Return to work

Distrust (low degree Insufficient

versus high degree)

Boudrez \&

De Backer $^{d}(19) \quad$ Positive Return to work

Perception job stress Insufficient

caused heart attack

Boudrez \&

De Backer ${ }^{d}(19) \quad$ Negative

Return to work 
Table 4. Continued.

\begin{tabular}{|c|c|c|c|}
\hline Factor & Result & Evidence & Outcome \\
\hline \multicolumn{2}{|c|}{ Perceived importance of work } & Moderate (+) & \\
\hline Boudrez et al (50) & Positive & & Return to work \\
\hline \multicolumn{4}{|c|}{ Wanting to go back to work } \\
\hline Samkange et al (47) & Positive & & Return to work \\
\hline \multicolumn{2}{|l|}{$\begin{array}{l}\text { Meaning of work versus } \\
\text { other aspects of life }\end{array}$} & Insufficient & \\
\hline Boudrez et al (50) & No effect & & Return to work \\
\hline \multicolumn{3}{|c|}{$\begin{array}{l}\text { Preoperative expectations regarding } \mathbf{S} \\
\text { work after surgery (yes versus no) }\end{array}$} & \\
\hline Engblom et al (49) & No effect & & Return to work \\
\hline Hlatky et al (31) & Positive & & Return to work \\
\hline $\begin{array}{l}\text { Boudrez \& } \\
\text { De Backer }{ }^{d}(19)\end{array}$ & Positive & & Return to work \\
\hline \multicolumn{2}{|c|}{ Satisfaction with sexuality } & Insufficient & \\
\hline Boudrez et al (50) & No effect ${ }^{b}$ & & Return to work \\
\hline Co-morbidity & & No evidence & \\
\hline Lundbom et al (27) & No effect ${ }^{b}$ & & $\begin{array}{l}\text { Working full- } \\
\text { time after CBS }\end{array}$ \\
\hline Hlatky et al (31) & No effect & & $\begin{array}{l}\text { Working full-- } \\
\text { time after CBS }\end{array}$ \\
\hline Caine et al (53) & No effect ${ }^{b}$ & & $\begin{array}{l}\text { Working full- } \\
\text { time after CBS }\end{array}$ \\
\hline \multicolumn{2}{|c|}{ Presence of diabetes (yes versus no) } & Moderate (-) & \\
\hline Hlatky et al (31) & No effect & & Return to work \\
\hline Drory et al (34) & Negative & & Return to work \\
\hline Froom et al (51) & Negative & & Return to work \\
\hline Mark et al (32) & No effect & & Return to work \\
\hline \multicolumn{2}{|c|}{ Previous health perception } & Insufficient & \\
\hline $\begin{array}{l}\text { Boudrez \& } \\
\text { De Backer }{ }^{\mathrm{e}}(19)\end{array}$ & No effect & & Return to work \\
\hline \multirow{2}{*}{$\begin{array}{l}\text { Attitude versus illness } \\
\text { Boudrez \& } \\
\text { De Backer }{ }^{d}(19)\end{array}$} & & Insufficient & \\
\hline & No effect & & Return to work \\
\hline \multirow{2}{*}{$\begin{array}{l}\text { Health concern } \\
\text { Soejima et al (45) }\end{array}$} & & Insufficient & \\
\hline & No effect & & Return to work \\
\hline \multirow{2}{*}{$\begin{array}{l}\text { Lack of perception of } \\
\text { stress-illness link } \\
\text { Soejima et al (45) }\end{array}$} & & Insufficient & \\
\hline & No effect & & Return to work \\
\hline
\end{tabular}

a Based on the best evidence synthesis level

${ }^{\mathrm{b}}$ Results based on univariate analysis.

c Indicated as numbers and percentages.

${ }^{\mathrm{d}}$ With ischemic heart disease related surgery.

${ }^{\mathrm{e}}$ Without ischemic heart disease related surgery.

f ST segment depression $>1 \mathrm{~mm}$ and abnormal SBP increase during exercise, indicated as numbers and percentages.

g $1-6=$ light versus $6=$ bad . start of the study (inception cohort) are not always well defined and may change in the course of the follow-up study. On the basis of this finding, we decided to use one category (ie, IHD). The only exception was the article of Boudrez (2007) (19), in which a difference was made in the analyses between patients with and without an operation. These two groups are described as two different cohorts.

\section{Results}

\section{Selection and methodological quality assessment}

The literature search resulted in 620 hits, of which 89 articles were marked "probable inclusion" or "indecisive result". The third reviewer decided on 11 articles, on which no consensus had been reached. Of the 89 articles, 48 were excluded. Reference checking yielded ten additional articles, three of which were included in this review, bringing the total to 44 studies: 20 for rheumatoid arthritis, two for asthma, one for asthma and COPD, 21 for IHD. All 44 articles were screened for quality; one low quality study on IHD was found (20) and excluded. In the final results, 43 studies were included, 32 of which were of high quality and 11 were of medium quality.

No cohort studies were found for diabetes mellitus. COPD was excluded from the results as a minimum of two articles is needed for inclusion and only one (21) was found. In this article, asthma and COPD were studied; consequently, only the results of the asthma patients were included in this review. Background information and the quality criteria for the selected cohort studies are presented in table 1. Data from the same cohort studies mentioned in more than one article have only been presented once in the final results. This applies to the articles of Reisine $(7,22)$ and Puolakka $(23,24)$. Chung et al (25) described two cohort studies (Nashville/Jyväskylä) in one article, but we presented the results as two studies. Also the results of the two cohorts of Boudrez et al (19) are described separately (IHD with and without surgery).

\section{Study characteristics}

The studies concerned different populations and countries, and did not use the same data sources nor define work disability or return to work in the same way. Puolakka defined work disability in two different ways. In one article (24), work disability is defined in terms of work disability days; in another (23), it is defined in terms of becoming a disability pensioner. Other authors have defined work disability in terms of losing of a full-time job or early retirement because of a chronic 
Table 5. Prognostic factors with sufficient evidence [weak ( $x)$, moderate $(\mathrm{Xx})$ or strong $(\mathrm{XxX})$ ] for work disability in rheumatoid arthritis (RA), asthma, and ischemic heart disease (IHD). Factors which increase (+) or decrease (-) the risk of work disability are indicated. The direction of the prognostic factors is presented as, for example, pain (high/low) which means a high pain condition versus a low one. (PTCA = purcutaneous transluminal coronary angioplasty, $\mathrm{AMI}=$ acute myocardial infarction).

\begin{tabular}{|c|c|c|c|c|c|c|}
\hline & \multicolumn{2}{|c|}{ RA } & \multicolumn{2}{|c|}{ Asthma } & \multicolumn{2}{|c|}{ IHD } \\
\hline & + & - & + & - & + & - \\
\hline \multicolumn{7}{|l|}{ Disease-related factors } \\
\hline Asthma severity score & & & $\mathrm{x}$ & & & \\
\hline Presence of congestive heart failure & & & & & $\mathrm{x}$ & \\
\hline Presence of angina pectoris & & & & & $\mathrm{x}$ & \\
\hline History of PTCA & & & & & & $x$ \\
\hline Complete revascularization (\%) & & & & & & $x$ \\
\hline \multicolumn{7}{|l|}{$\begin{array}{l}\text { Impairments in body functions/ } \\
\text { body structure }\end{array}$} \\
\hline Presence of reumatoid factor & $x x$ & & & & & \\
\hline Erythrocyte sedimentation rate (ESR) & $\mathrm{x}$ & & & & & \\
\hline Amount of damaged joints & $x x$ & & & & & \\
\hline Amount of swollen joints & $x x$ & & & & & \\
\hline Pain (high / low) & $x x$ & & & & & \\
\hline Fatigue (high / low) & $x$ & & & & & \\
\hline Presence of dyspnoea (no / yes) & & & & & & $x x$ \\
\hline Chest pain (yes / no) & & & & & $x x$ & \\
\hline Left ventricular function (normal / abnormal) & & & & & & $x x$ \\
\hline Peak workflow (watts) & & & & & & $x$ \\
\hline Presence of depression & & & & & $x x x$ & \\
\hline Somatic complaints (high / low) & & & & & $x x x$ & \\
\hline$Q$ wave AMI (yes / no) & & & & & $x x$ & \\
\hline Summary psychological scales & & & & & & $\mathrm{x}$ \\
\hline \multicolumn{7}{|l|}{ Activity limitations and participation restrictions } \\
\hline Health Assessment Questionnaire (>1.5) & $x \mathrm{xx}$ & & & & & \\
\hline Sickness absence & $\mathrm{x}$ & & & & & \\
\hline Duke activity scale & & & & & $\mathrm{x}$ & \\
\hline Subjective vocational disability (strong / light & & & & & $x x x$ & \\
\hline Sick leave before operation (yes / no) & & & & & $x x x$ & \\
\hline \multicolumn{7}{|l|}{ Environmental factors } \\
\hline Occupation type (blue- versus white-collar) & $x x x$ & & $x x$ & & $x x$ & \\
\hline Precision work (high / low) & $x \mathrm{x}$ & & & & & \\
\hline Non-sedentary work / sedentary work & $x$ & & & & & \\
\hline Working hours (full-time / part-time) & $\mathrm{x}$ & & & & & \\
\hline Data complexity at work (high / low) & $\mathrm{x}$ & & & & & \\
\hline Residence (rural / urban) & & & & & $x x$ & \\
\hline Attended cardiac rehabilitation (yes / no) & & & & & & $x x$ \\
\hline Ever/ never used methotrexate & $\mathrm{x}$ & & & & & \\
\hline Delay to treatment (early / late) & & $\mathrm{x}$ & & & & \\
\hline Ever/never used prednisone & $\mathrm{x}$ & & & & & \\
\hline Support from friends (yes / no) & & & & & & $\mathrm{x}$ \\
\hline Health insurance & & & & & & $\mathrm{x}$ \\
\hline \multicolumn{7}{|l|}{ Personal factors } \\
\hline Age (high / low) & $x x x$ & & & & $x x x$ & \\
\hline Age at onset $(>50$ years $/<50$ years) & $x$ & & & & & \\
\hline Gender (female / male) & $x x$ & & $\mathrm{x}$ & & $\mathrm{x}$ & \\
\hline Education (high / low) & & $x x$ & & & & $x x x$ \\
\hline Socioeconomic status (high / low) & & & & & & $x$ \\
\hline Ethnic origin (white / non-white) & & $x x$ & & & $\mathrm{x}$ & \\
\hline Patient global severity score & $x x$ & & & & & \\
\hline Desire to remain employed (yes / no) & & $\mathrm{x}$ & & & & \\
\hline Preoperative expectations regarding & & & & & & \\
\hline work after surgery (yes / no) & & & & & & $x x x$ \\
\hline Perceived importance of work / & & $x$ & & & & $x x$ \\
\hline Satisfaction with working & & & & & & \\
\hline conditions (yes / no) & & $\mathrm{x}$ & & & & \\
\hline Presence of diabetes (yes / no) & & & & & $x x$ & \\
\hline Health locus of control (internal / external) & & & & & & $x x$ \\
\hline
\end{tabular}

disease (26). There were also variations in the definition of return-to-work employees with IHD. Some authors $(27,28)$ used the definition of return to work as working (full-time) after coronary bypass surgery, while others $(29,30)$ defined it as the resumption of a former job or starting a new job, on a full- or part-time basis.

\section{Prognostic factors for work disability}

The prognostic factors found in the literature for rheumatoid arthritis, asthma, and IHD are presented in tables 2-4 including the original outcomes mentioned in the articles (ie, work disability, return to work, etc). To facilitate the interpretation and comparison of the results, in table 5 and the following sections, we have synthesized the results for the outcome work disability only. Table 5 presents all the prognostic factors supported by weak, moderate, or strong evidence for each disease for the outcome work disability.

Disease-related factors. For employees with rheumatoid arthritis, there is currently not enough evidence on disease-related factors for any conclusion to be drawn. Among employees with asthma, the asthma severity score has been found to have a weak positive relation with work disability. One study found a positive relation between the asthma severity score and work disability (10) while another study found no such relation at all (11).

In the case of employees with $I H D$, a weak negative relation has been found between the presence of congestive heart failure and return to work: one article found no relation (31); one article found a negative relation (32). The presence of angina pectoris has been found to be a weak positive factor of work disability: one article found a positive relation (33); another article found no relation (32). Two factors, a history of PTCA and a complete revascularization, have been found to have a weak negative relation for work disability. For PTCA, one article found a negative relation (34); one found no effect (33). For a complete revascularization, one study found a positive relation (19); one study found no effect (33).

Impairments in body function or body structure. In employees with rheumatoid arthritis, various factors related to impaired body function or body structures have been found to be predictive of work disability. A moderate positive relation has been found between the presence of a rheumatoid factor $(\mathrm{RF}+)$ and work disability: four studies found a positive relation (35-38) and four studies found no relation $(24,25,39-40)$. The amount of deformed and swollen joints has been found to be a moderate positive prognostic factor for work disability: three studies found a positive relation between the amount of deformed joints and work disability (22, $35,41)$, and four studies found no effect $(7,24,26,42)$. 
Four studies found a positive relation between the amount of swollen joints and work disability $(22,35$, $40,41)$, and six studies found no relation $(24,36,39$, 42-44). A moderate positive effect has been found for pain: four studies were positive $(8,18,36,37)$ and six showed no effect $(7 / 22,18,24,42-44)$. Weak evidence has been found for the erythrocyte sedimentation rate and fatigue as prognostic factors of work disability in rheumatoid arthritis.

For employees with asthma, there is currently not enough evidence on factors which are predictive of work disability in this category.

The presence of depression and the level of somatic complaints (high versus low) have been found to be strong positive prognostic factors of work disability for employees with $I H D$. Six studies found a positive relation $(30,45-49)$ and one article found no relation (49) between depression and work disability. The level of somatic complaints was found to have a positive relation with work disability in three articles $(19,28,50)$, while two articles found no effect $(19,46)$. For employees with IHD, Q wave in cases with acute myocardial infarction (yes versus no) $(34,51)$ and chest pain (yes versus no) $(32,52,53)$ were found to be moderate positive prognostic factors for work disability. The presence of dyspnea (no versus yes) $(33,53)$ and left ventricular function (normal versus abnormal) $(19,31)$ have been shown to be moderate negative prognostic factors for work disability. Weak negative factors that have been found include peak/final workflow $(33,49)$ and a sum score of psychological problems $(19,32)$.

To conclude, we did not find any studies which identified common prognostic factors valid for more than one disease in this category.

Activity limitations and participation restrictions. For employees with rheumatoid arthritis, two factors have been found to be predictive of work disability: a high score on the health assessment questionnaire (HAQ) (strong positive) and previous sickness absence (weak positive). Eight studies found a positive relation between a high score on the HAQ and work disability (23/24, $26,39,42-44,54,55)$. Two studies found no relation $(25,36)$.

In employees with asthma, there was not enough evidence on any factors in this category for any conclusion to be drawn.

For employees with $I H D$, sickness absence before operation has been found to be a strong positive prognostic factor of work disability: five articles found a positive relation with work disability $(19,27-29,53)$ and one article found no relation (49). The patient's view on the limitations caused by the disease at work (strong versus light) has been found to be another strong positive prognostic factor for work disability in employees with IHD: two articles found a positive relation with work disability $(47,56)$, and one article found no relation (49). Functional activity (the patient's view) has been found to be a weak positive factor of work disability: one article found a positive relation (32) and the other no relation at all (31).

Based on the evidence, we can conclude that the limitation in daily activities caused by the disease (HAQ and Duke activity scale) and sickness absence (for IHD before operation) are common prognostic factors for rheumatoid arthritis and IHD.

Environmental factors. The type of employment (heavy manual work/blue-collar work has been shown to be a strong positive prognostic factor for work disability in rheumatoid arthritis. Six studies reported a positive relation with work disability $(7,26,36,40,44,57)$ and four studies found no effect $(24,40-42)$. Another moderate positive prognostic factor found in the literature was precision versus non-precision work $(35,43)$. Studies have shown weak positive factors to include the following: non-sedentary versus sedentary work (25), complex work (high versus low) $(22,36)$, working hours (part-time versus full-time) $(7,22,57)$, and the use of methotrexate and prednisone (25). Another weak negative prognostic factor found in the literature was the commencement of medication use $(<4$ months versus $>4$ months) $(24,25)$.

For employees with asthma, heavy manual work/ blue-collar work was reported to be a moderate positive factor of work disability $(11,21)$.

Studies have also shown heavy manual work/bluecollar work to be a moderate positive prognostic factor of work disability for employees with $I H D$. Four articles have shown a positive relation between work disability and heavy manual work/blue-collar work $(19,33,34$, 47), while six articles found no relation between the type of occupation and work disability $(27,31,45,49,50$, 53). Living in a rural versus urban area was identified in the literature as another moderate positive factor for work disability $(27,33,52)$; the attendance of a cardiac rehabilitation program was found to be a moderate negative factor $(50,58)$. Support from friends (yes versus no) and health insurance were shown to be weak negative prognostic factors for work disability $(19,45,50$, and 31,58 , respectively).

In the category "environmental factors", we can conclude that the type of employment (manual/bluecollar work versus non-manual/white-collar work) is a prognostic factor that results in a higher risk of work disability in all diseases.

Personal factors. Studies have shown that age (high versus low) has been found to be a strong positive prognostic factor of work disability for employees with 
rheumatoid arthritis. Ten studies reported a positive relation $(7,22-25,35,37,40,43,57)$, while four studies found no relation $(25,38,44,54)$. A moderate positive relation has been shown between gender (female versus male) and work disability. Five studies found a positive relation $(26,36,38,54,57)$ and ten studies found no relation at all $(7,22,25,37,39,40,42-44)$. The literature has also shown the patient global severity score (high versus low) to be a moderate positive factor $(24,25,36)$. Education (high versus low) has been shown to be a moderate negative prognostic factor of work disability in rheumatoid arthritis. Six studies reported a negative relation $(25,36,37,42,54,57)$ and seven studies found no relation $(7,22,24,39,40,43$, 44). Ethnic origin (white versus non-white) has also been identified as a moderate negative factor for work disability $(25,36,41)$. The literature has identified age at the onset of the disease to be a weak positive prognostic factor $(26,39)$; weak negative prognostic factors include the desire to remain employed (yes versus no) and satisfaction with working conditions $(7,22$, and 41 , 42, 44 respectively).

For employees with asthma, gender (female versus male) was found to have a weak negative relation with continuous employment and thus a weak positive relation with work disability. One study reported a negative relation (11) while another found no effect (10).

Age (high versus low) was shown to be a strong positive factor of work disability for employees with $I H D$. A positive relation between an older age and work disability was reported in 14 studies $(27-29,31-34$, $46-48,50-52,56$ ), while three studies found no relation $(30,45,53)$. The literature has identified education (high versus low) as being a strong negative factor of work disability. Three studies report a negative relation (32, 48,58 ); one study found no relation (45). The patient's expectation (yes versus no) regarding return to work after a coronary bypass operation was reported to be a strong negative prognostic factor of work disability. Two articles showed a negative relation $(19,31)$ while a third found no relation (49). An internal locus of health control in patients with IHD has also been found to have a moderate negative relation with work disability. One study reported a positive relation with return to work (46), while three studies found no relation $(19,45,58)$. The perceived importance of work (high versus low) was identified as a moderate negative factor $(47,50)$ and the presence of diabetes (yes versus no) a moderate positive factor for work disability $(31,32,34,51)$. A weak positive relation has been shown between gender (female versus male) and work disability $(30-34,47)$, while a weak negative relation was found between ethnic origin (white versus non-white), socioeconomic status (high versus low) and work disability (32, 58 and 46,49 , respectively).
To summarize, in the category "personal factors", gender (female) has been found to be a common positive determinant of work disability for asthma, rheumatoid arthritis and IHD. Being of older age has been identified as a strong positive prognostic factor of work disability in rheumatoid arthritis and IHD. Furthermore, education (high versus low) and ethnic origin (white versus non-white) have been shown to be negative prognostic factors for work disability in employees with rheumatoid arthritis and IHD.

\section{Discussion}

\section{Relevant factors}

Using prospective and retrospective disease cohort studies, the aim of this study was to identify common prognostic or risk factors for work disability in employees with rheumatoid arthritis, asthma, COPD, IHD, and diabetes mellitus. We found only one cohort study for COPD and none for diabetes mellitus. We did, however, identify a number of studies which point to common prognostic factors for rheumatoid arthritis, asthma and IHD.

In the category "impaired body function or body structure", studies have identified many prognostic factors. For rheumatoid arthritis and IHD, the perceived health complaints were mostly related to somatic complaints. In IHD, depression was also found to be a positive prognostic factor of work disability. In the category "activity limitations", studies examining rheumatoid arthritis and IHD have found that disease-related restrictions in daily physical activities (high versus low) is a positive prognostic factor for work disability. Studies have also identified sickness absence as a common prognostic factor between rheumatoid arthritis and IHD. In the category "environmental factors", studies have pointed to the type of employment (ie, manual work/ blue-collar work versus non-manual work/white-collar work) as a prognostic factor that results in a higher risk of work disability in all diseases. While studies have found social support at work to be a prognostic factor of work disability in IHD, no evidence has been found for rheumatoid arthritis or asthma.

In the category "personal factors", studies have identified several common factors: being of an older age is a strong prognostic factor for work disability in all three diseases. Female gender is a strong positive prognostic factor in employees with rheumatoid arthritis and asthma. High education is a negative prognostic factor for work disability in employees with rheumatoid arthritis and IHD. In the latter, an internal health locus of control and perceived importance of wanting to 
work have been found to be a negative factor for work disability. For employees with rheumatoid arthritis and asthma, we did not find any cohort studies identifying the prognostic factors of health locus of control or coping with the illness. In rheumatoid arthritis, studies have found the desire to remain employed to be a negative prognostic factor of work disability. Factors such as coping with disease symptoms and health locus of control are possibly important for continuing to work or return-to-work intervention programs but they have not yet been analyzed in observational cohort studies for all the diseases. This review confirms the prognostic factors found in rheumatoid arthritis for work disability in other reviews $(17,59)$. We did not find any other reviews pointing to prognostic factors for work disability in asthma, IHD, and diabetes mellitus.

Most risk factors with weak, moderate or strong evidence found in this systematic review were disease/impairment-related, socio-demographic-related, or work-related. Common prognostic factors that we found in the literature were: (i) various severity of disease/ impairment factors, (ii) gender (female versus male), (iii) age (high versus low), (iv) education (low versus high), (v) heavy manual work (blue- versus white-collar work), (vi) sick leave, and (vii) perceived health complaints (high versus low). Factors such as low functional status, age, gender, education and blue-collar work are already known to be risk factors for work disability for employees without a chronic disease $(60,61)$.

A serious weakness of most of the cohort studies reviewed was that they had not analyzed the role of psychosocial personal factors (eg, health locus of control or coping). Cross-sectional studies have suggested that there is a causal relationship between low psychological well-being and high health-related distress, and an increase in work limitations among employees with various chronic diseases (62-64). A cross-sectional study (65) has also identified an association between psychological distress, an external health locus of control, and reported disabilities in patients with lower back pain. Working women, non-whites, and low-educated employees suffer more psychological distress because of a chronic medical condition $(66,67)$. This finding may partly explain the excessive risk of work disability in women and low-educated, blue-collar workers.

A well-known pathway is the relationship between coping style and the health locus of control on one side, and gender, education, age, and blue-collar work on the other. A meta-analysis of Tamres et al (68) showed that, compared to men, women are more likely to use an external rather than internal health locus of control (ie, have a passive versus active coping style). A study of van der Linden et al (69) showed that there is an association between low education, a higher age, blue-collar work and an external health locus of control.
More research is needed on the influence of workrelated and psychosocial personal factors on work disability as such factors can be influenced by work-oriented behavioral and environmental interventions. Supporting employees at work to cope with the limitations of the disease (in terms of self management behavior at work) has been found to be associated with lower psychological distress and a greater control over the disease. These employees are also more likely to be proactive in seeking support at work (70-71). Active coping has also been shown to reduce the chance of work disability (72). One article on IHD (28) found a positive relation between return to work and coping with or accepting the illness (ie, active versus passive sick role).

\section{Methodological problems}

We encountered five methodological problems in this systematic review. Firstly, there were methodological differences between the studies: work-related outcomes and definitions of work disability were different, measurements were taken at different times, and definitions of prognostic variables (eg, various age categories and cut-off points) were not standardized. Of paramount importance is the fact that national differences in the organization of the social security system and unemployment rates are major determinants of work disability risks. These overarching differences can also have an impact on the influence of prognostic factors, which has to be taken into account in the interpretation of findings and the implementation of measures.

Secondly, it was difficult to find cohort studies on asthma, COPD, and diabetes mellitus. For diabetes mellitus, we found only cross-sectional studies providing "determinants" of the relation between the disease and work disability. The determinants found were similar to the prognostic factors identified in the cohort studies in the present study, for example, age (high versus low) and gender (female versus male), both of which were positively associated with work disability, and education (high versus low) which was found to be negatively associated with work disability (73-75).

Thirdly, the results of the review were based on the evidence provided in the cohort studies. This may imply that some variables, which in occupational practice are supposed to have an effect on work disability, may not have been analyzed in the studies. An example of this is the lack of data regarding the prognostic factors for work disability in asthma. More research is needed on the effects of environmental and occupational exposure as these factors are known to be related to work disability in employees with asthma.

Fourthly, we decided in this review to give priority to data from multivariate analysis over data from univariate analysis as the former controls for confounding 
variables like gender and age (76) - particularly as controlling for confounding is included in international guidelines as an important criterion to screen the quality of an observational study $(77,78)$. Nevertheless, a disadvantage of multivariate analysis is the difficulty faced by or the subjectivity of the researchers when constructing adequate models. In articles, information is not always presented on the intercorrelations between the independent factors involved and whether one factor overrides the other in the final model. An example is the forced expiratory volume (FEV\%) and the asthma severity score in employees with asthma - two prognostic variables that highly correlate with each other (10). Multivariate analysis can select one variable to the exclusion of another when there may be only minor differences in the strength of their prediction. In the final model, the researcher may decide to exclude or to keep one of the variables. In univariate analysis, more factors are visible as having a relation with work disability but an important disadvantage is that confounding and effect modifying is invisible.

Finally, due to the inclusion of a variety of chronic diseases and the wide range of prognostic variables found, it was not possible to effectuate a meta-analysis (79). In order to overcome the limitations of the methods of a traditional narrative review and a meta-analysis, we used the method of "best-evidence synthesis" (16). This method combines the strengths of the methods of both traditional narrative review and meta-analysis, incorporating the statistical rigor of the latter to synthesize quantitative findings as well as the flexibility of the former. Subjectivity in this method is, to a certain extent, excluded by the use of well-justified and well-described inclusion criteria for the studies (16).

\section{Recommendations for further research}

From the findings in this study, we derived some recommendations for further research.

Firstly, more observational cohort studies with objective data on the effect of coping style and health locus of control on work disability are needed, as these variables can be influenced by work-oriented behavioral interventions. Secondly, more observational cohort studies are needed on the effect of work factors on work disability, as these factors can also be influenced by interventions at the workplace. Thirdly, more observational cohort studies in general are needed for COPD, asthma, and diabetes mellitus. Fourthly, more research and discussion is needed on the advantages and disadvantages of different prognostic models as data may be lost by using a final multivariate model of analysis. A combination of strategies may provide more transparency, better insights of data, and more efficient control of confounding.

\section{Concluding remarks}

This review shows that several prognostic factors for work disability have been found to be common amongst employees with a chronic physical disease. This implies that these prognostic or risk factors have a more generic character beside the more specific problems for working life that may be related to every chronic disease. The common factors are presumably related to the impact of a long-lasting or recurrent health problem on working life as such. As many prognostic factors for work disability are common, we are now better equipped to distinguish groups of employees at high risk of becoming work disabled, regardless of the employee's specific disease. In short, we have to pay special attention to (i) employees with a more severe chronic disease, including a high level of perceived health complaints, (ii) employees with disease-specific impaired body functions, such as pain and swollen/deformed joints in rheumatoid arthritis and depression in IHD, and (iii) employees with more daily physical limitations caused by the disease. Special consideration of older workers, women, bluecollar workers and low-educated employees is also needed. We may be able to use this information in the development and implementation of general disability management interventions for employees with a chronic disease to overcome health-related limitations at work.

\section{Acknowledgements}

This study was financially supported by the HAN University of Applied Sciences in the Netherlands. The authors wish to acknowledge the contribution of Tjeerd de Jong and Frank Stöteler, HAN University of Applied Sciences, Hans Groenewoud, Department of Epidemiology and Biostatistics, Radboud University Nijmegen Medical Centre, and Jaap van Dijk and Jan Hoving, Coronel Institute of Occupational Health, Academic Medical Center, University of Amsterdam.

\section{References}

1. Dupre D, Karjalainen A. Eurostat, statistics in focus: employment of disabled people in Europe in 2002, Eurostat, theme 3: population and social conditions. Brussels: Statistical Office of the European Communities; 2003.

2. Blokstra A, Baan CA, Boshuizen HC, Feenstra TL, Hoogenveen RT, Picavet HS, et al. Impact of the ageing population on burden of disease: projections of chronic disease prevalence for 2005-2025. Bilthoven (the Netherlands): National Institute for Public Health and Environment; 2007. 
3. Druss BG, Marcus SC, Olfson M, Tanielian T, Elinson L, Pincus HA. Comparing the national economic burden of five chronic conditions. Health Aff. 2001;20(6):233-41.

4. Netherlands Institute for Health Services Research. National Panel of the Chronically Ill and Disabled. Utrecht (the Netherlands): Netherlands Institute for Health Services Research; 2005.

5. Pascual M, Cantarero D. Socio-demographic determinants of disabled people: an empirical approach based on the European Community Household Panel. J Socio Econ. 2007;36(2):275-87.

6. Allaire SH, Anderson JJ, Meenan R. Reducing work disability associated with rheumatoid arthritis: identification of additional risk factors and persons likely to benefit from intervention. Arthritis Care Res. 1996;9(5):349-57.

7. Reisine S, Fifield J, Walsh SJ, Feinn R. Factors associated with continued employment among patients with rheumatoid arthritis: a survival model. J Rheumatol. 2001;28(11):2400-8.

8. Mancuso CA, Rincon M, Sayles W, Paget SA. Longitudinal study of negative workplace events among employed rheumatoid arthritis patients and healthy controls. Arthritis Rheum. 2005;15;53(6):958-64.

9. Eisner MD, Yelin EH, Katz PP, Lactao G, Iribarren C, Blanc PD. Risk factors for work disability in severe adult asthma. Am J Med. 2006;119(10):884-91.

10. Blanc PD, Yen IH, Chen H, Katz PP, Earnest G, Balmes J, et al. Area-level socio-economic status and health status among adults with asthma and rhinitis. Eur Respir J. 2006;27(1):85-94.

11. Yelin E, Katz P, Balmes J, Trupin L, Earnest G, Eisner M, et al. Work life of persons with asthma, rhinitis, and COPD: a study using a national, population-based sample. J Occup Med Toxicol. 2006;2;2.

12. Detaille SI, Haafkens JA, van Dijk FJ. What employees with rheumatoid arthritis, diabetes mellitus and hearing loss need to cope at work. Scand J Work Environ Health. 2003;29(2):134-42.

13. Poos MJJC (RIVM). Which diseases have the highest prevalence? In: Volksgezondheid Toekomst Verkenning. Nationaal Kompas Volksgezondheid. [Dutch Public Health Status and Forecasts Report, National Institute for Public Health and the Environment]; 2006

14. The Dutch Cochrane Center. Amsterdam: Academic Medical Center; 2008. www.cochrane.nl

15. World Health Organization (WHO). International Classification of Functioning, Disability and Health (ICF). Geneva: WHO; 2001.

16. Slavin RE. Best evidence synthesis: an intelligent alternative to meta-analysis. J Clin Epidemiol. 1995;48(1):9-18.

17. de Croon EM, Sluiter JK, Nijssen TF, Dijkmans BA, Lankhorst GJ, Frings-Dresen MH. Predictive factors of work disability in rheumatoid arthritis: a systematic literature review. Ann Rheum Dis. 2004;63(11):1362-7.

18. Steenstra IA, Verbeek JH, Heymans MW, Bongers PM. Prognostic factors for duration of sick leave in patients sick listed with acute low back pain: a systematic review of the literature. Occup Environ Med. 2005;62(12):851-60.
19. Boudrez H, De Backer G. Recent findings on return to work after an acute myocardial infarction or coronary artery bypass grafting. Acta Cardiol. 2000;55(6):341-9.

20. Abbas AE, Brodie B, Stone G, Cox D, Berman A, Brewington $\mathrm{S}$, et al. Frequency of returning to work one and six months following percutaneous coronary intervention for acute myocardial infarction. Am J Cardiol. 2004;94(11):1403-5.

21. Alexopoulos EC, Burdorf A. Prognostic factors for respiratory sickness absence and return to work among blue collar workers and office personnel. Occup Environ Med. 2001;58(4):246-52.

22. Reisine S, McQuillan J, Fifield J. Predictors of work disability in rheumatoid arthritis patients: a five-year followup. Arthritis Rheum. 1995;38(11):1630-7.

23. Puolakka K, Kautiainen H, Pekurinen M, Möttönen T, Hannonen P, Korpela M, et al. Monetary value of lost productivity over a five year follow up in early rheumatoid arthritis estimated on the basis of official register data on patients' sickness absence and gross income: experience from the FIN-RACo trial. Ann Rheum Dis. 2006;65(7):899-904.

24. Puolakka K, Kautiainen H, Möttönen T, Hannonen P, Hakala M, Korpela M, et al. Predictors of productivity loss in early rheumatoid arthritis: a 5 year follow up study. Ann Rheum Dis. 2005;64(1):130-3.

25. Chung CP, Sokka T, Arbogast PG, Pincus T. Work disability in early rheumatoid arthritis: higher rates but better clinical status in Finland compared with the US. Ann Rheum Dis. 2006;65(12):1653-7.

26. Young A, Dixey J, Kulinskaya E, Cox N, Davies P, Devlin J, et al. Which patients stop working because of rheumatoid arthritis?: results of five years' follow up in 732 patients from the Early RA Study (ERAS). Ann Rheum Dis. 2002;61(4):335-40.

27. Lundbom J, Myhre HO, Ystgaard B, Bolz KD, Hammervold R, Levang OW. Factors influencing return to work after aortocoronary bypass surgery. Scand J Thorac Cardiovasc Surg. 1992;26(3):187-92.

28. Agren B, Rydén O, Johnsson P, Nilsson-Ehle P. Rehabilitation after coronary bypass surgery: coping strategies predict metabolic improvement and return to work. Scand J Rehabil Med. 1993;25(2):83-95.

29. Munro WS. Work before and after coronary artery bypass grafting. J Soc Occup Med. 1990;40(2):59-64.

30. Bhattacharyya MR, Perkins-Porras L, Whitehead DL, Steptoe A. Psychological and clinical predictors of return to work after acute coronary syndrome. Eur Heart J. 2007;28(2):160-5.

31. Hlatky MA, Haney T, Barefoot JC, Califf RM, Mark DB, Pryor DB, et al. Medical, psychological and social correlates of work disability among men with coronary artery disease. Am J Cardiol. 1986;58(10):911-5.

32. Mark DB, Lam LC, Lee KL, Clapp-Channing NE, Williams $\mathrm{RB}$, Pryor DB, et al. Identification of patients with coronary disease at high risk for loss of employment: a prospective validation study. Circulation. 1992;86(5):1485-94.

33. Sellier P, Varaillac P, Chatellier G, D'Agrosa-Boiteux MC, Douard $\mathrm{H}$, Dubois $\mathrm{C}$, et al. Factors influencing return to work at one year after coronary bypass graft surgery: results 
of the PERISCOP study. Eur J Cardiovasc Prev Rehabil. 2003;10(6):469-75.

34. Drory Y, Kravetz S, Koren-Morag N, Goldbourt U. Resumption and maintenance of employment after a first acute myocardial infarction: sociodemographic, vocational and medical predictors. Cardiology. 2005;103(1):37-43.

35. Mau W, Bornmann M, Weber H, Weidemann HF, Hecker H, Raspe HH. Prediction of permanent work disability in a followup study of early rheumatoid arthritis: results of a tree structured analysis using RECPAM. Br J Rheumatol. 1996;35(7):652-9.

36. Wolfe F, Hawley DJ. The longterm outcomes of rheumatoid arthritis: work disability: a prospective 18 year study of 823 patients. J Rheumatol. 1998;25(11):2108-17.

37. Straaton KV, Maisiak R, Wrigley JM, White MB, Johnson $\mathrm{P}$, Fine PR. Barriers to return to work among persons unemployed due to arthritis and musculoskeletal disorders. Arthritis Rheum. 1996; 39(1):101-9.

38. Albers JM, Kuper HH, van Riel PL, Prevoo ML, van 't Hof MA, van Gestel AM, et al. Socio-economic consequences of rheumatoid arthritis in the first years of the disease. Rheumatology (Oxford). 1999;38(5):423-30.

39. Barrett EM, Scott DG, Wiles NJ, Symmons DP. The impact of rheumatoid arthritis on employment status in the early years of disease: a UK community-based study. Rheumatology (Oxford). 2000;39(12):1403-9.

40. Sokka T, Kautiainen H, Möttönen T, Hannonen P. Work disability in rheumatoid arthritis 10 years after the diagnosis. J Rheumatol. 1999;26(8):1681-5.

41. Reisine S, Fifield J, Walsh S, Dauser D. Work disability among two cohorts of women with recent-onset rheumatoid arthritis: a survival analysis. Arthritis Rheum. 2007;57(3):372-80.

42. Fex E, Larsson BM, Nived K, Eberhardt K. Effect of rheumatoid arthritis on work status and social and leisure time activities in patients followed 8 years from onset. J Rheumatol. 1998;25(1):44-50.

43. Borg G, Allander E, Berg E, Brodin U, From A, Trang L. Auranofin treatment in early rheumatoid arthritis may postpone early retirement: results from a 2-year double blind trial. J Rheumatol. 1991;18(7):1015-20.

44. Eberhardt KB, Fex E. Functional impairment and disability in early rheumatoid arthritis-development over 5 years. J Rheumatol. 1995;22(6):1037-42.

45. Soejima Y, Steptoe A, Nozoe S, Tei C. Psychosocial and clinical factors predicting resumption of work following acute myocardial infarction in Japanese men. Int J Cardiol. 1999;72(1):39-47.

46. Abbott J, Berry N. Return to work during the year following first myocardial infarction. Br J Clin Psychol. 1991;30 (Pt 3):268-70.

47. Samkange-Zeeb F, Altenhöner T, Berg G, Schott T. Predicting non-return to work in patients attending cardiac rehabilitation. Int J Rehabil Res. 2006;29(1):43-9.

48. Söderman E, Lisspers J, Sundin O. Depression as a predictor of return to work in patients with coronary artery disease. Soc Sci Med. 2003;56(1):193-202.
49. Engblom E, Hämäläinen $H$, Rönnemaa T, Vänttinen E, Kallio V, Knuts LR. Cardiac rehabilitation and return to work after coronary artery bypass surgery. Qual Life Res. 1994;3(3):207-13.

50. Boudrez H, De Backer G, Comhaire B. Return to work after myocardial infarction: results of a longitudinal population based study. Eur Heart J. 1994;15(1):32-6.

51. Froom P, Cohen C, Rashcupkin J, Kristal-Boneh E, Melamed $\mathrm{S}$, Benbassat J, et al. Referral to occupational medicine clinics and resumption of employment after myocardial infarction. J Occup Environ Med. 1999;41(11):943-7.

52. Speziale G, Bilotta F, Ruvolo G, Fattouch K, Marino B. Return to work and quality of life measurement in coronary artery bypass grafting. Eur J Cardiothorac Surg. 1996;10(10):852-8.

53. Caine N, Harrison SC, Sharples LD. Wallwork J. Prospective study of quality of life before and after coronary artery bypass grafting. BMJ. 1991;302(6775):511-6.

54. Odegard S, Finset A, Kvien TK, Mowinckel P, Uhlig T. Work disability in rheumatoid arthritis is predicted by physical and psychological health status: a 7-year study from the Oslo rheumatoid arthritis register. Scand J Rheumatol. 2005;34(6):441-7

55. Jäntti J, Aho K, Kaarela K, Kautiainen H. Work disability in an inception cohort of patients with seropositive rheumatoid arthritis: a 20 year study. Rheumatology (Oxford). 1999;38(11):1138-41.

56. Mittag O, Kolenda KD, Nordman KJ, Bernien J, Maurischat C. Return to work after myocardial infarction/coronary artery bypass grafting: patients' and physicians' initial viewpoints and outcome 12 months later. Soc Sci Med. 2001;52(9):1441-50.

57. Holte HH, Tambs K, Bjerkedal T. Becoming a disability pensioner with rheumatoid arthritis in Norway 1971-1990. J Rheumatol. 2001;28(1):54-61.

58. Earle A, Ayanian JZ, Heymann J. Work resumption after newly diagnosed coronary heart disease: findings on the importance of paid leave. J Womens Health (Larchmt). 2006;15(4):430-41.

59. Burton W, Morrison A, Maclean R, Ruderman E. Systematic review of studies of productivity loss due to rheumatoid arthritis. Occup Med. 2006;56(1):18-27.

60. Randolph DS. Predicting the effect of disability on employment status and income. Work. 2004;23(3):257-66.

61. Kampfe CM, Wadsworth JS, Mamboleo GI, Schonbrun SL. Aging, disability, and employment. Work. 2008;31(3):337-44.

62. Munir F, Yarker J, Haslam C, Long H, Leka S, Griffiths A, et al. Work factors related to psychological and health-related distress among employees with chronic illnesses J Occup Rehabil. 2007;17(2):259-77.

63. Akabas SH, Gates LB. Stress and disability management project. New York (NY): Center for Social Policy and Practice in the workplace, Columbia University; 1993. Report for The National Institute on Disability and Rehabilitation Research.

64. Boot CR, Heijmans M, van der Gulden JW, Rijken M. The role of illness perceptions in labour participation of the chronically ill. Int Arch Occup Environ Health. 2008;82(1):13-20. 
65. Härkäpää K. Relationships of psychological distress and health locus of control beliefs with the use of cognitive and behavioral coping strategies in low back pain patients Clin J Pain. 1991;7(4):275-82.

66. Curtis R, Groarke A, Coughlan R, Gsel A. Psychological distress as a predictor of psychological adjustment and health status in patients with rheumatoid arthritis. Patient Educ Couns. 2005;59:192-8.

67. Maes S, Leventhal H, Ridder DTD. Coping with chronic diseases. In: Zeidner M, Endler N, editors. Handbook of coping: theory, research and applications. New York (NY): Wiley; 1996. p 221-51.

68. Tamres LK, Janicki D, Helgeson VS. Sex differences in coping behavior: a meta-analytic review and an examination of relative coping. Pers Soc Psychol Rev. 2002;6(1):2-30.

69. Van der Linden M, van den Akker M, Buntinx F. The relation between health locus of control and multimorbidity: a case control study. Pers Individ Dif. 2001;30:1189-97.

70. Munir F, Leka S, Griffiths A. Dealing with self-management of chronic illness at work: predictors for self-disclosure. Soc Sci Med. 2005;60:1397-407.

71. Van Rhenen W, Schaufeli W, Van Dijk F, Blonk R. Coping and sickness absence. Int Arch Occup Environ Health. 2008;81:461-72.

72. Pool G, Heuvel F, Ranchor A. Handboek psychologische interventies bij chronisch-somatische aandoeningen [Guideline of psychological interventions for people with a chronic somatic disease]. Assen (the Netherlands): Koninklijke van Gorkum; 2004.

73. Mayfield JA, Deb P, Whitecotton L. Work disability and diabetes. Diabetes Care. 1999;22(7):1105-9.

74. Von Korff M, Katon W, Lin EH, Simon G, Ciechanowski $\mathrm{P}$, Ludman E, et al. Work disability among individuals with diabetes. Diabetes Care. 2005;28(6):1326-32.

75. Tunceli K, Bradley CJ, Nerenz D, Williams LK, Pladevall M, Elston Lafata J. The impact of diabetes on employment and work productivity. Diabetes Care. 2005;28(11):2662-7.

76. Hernberg S. Introduction to occupational epidemiology. Chelsea (MI): Lewis Publishers; 1992.

77. Hayden JA, Côté P, Bombardier C. Evaluation of the quality of prognosis studies in systematic reviews. Ann Intern Med. 2006;144(6):427-37

78. von Elm E, Altman DG, Egger M, Pocock SJ, Gøtzsche PC, Vandenbroucke JP, et al. The Strengthening the Reporting of Observational Studies in Epidemiology (STROBE) statement: guidelines for reporting observational studies. PLoS Med. 2007;4(10):e296.

79. Rosenthal R, DiMatteo MR. Meta-analysis: recent developments in quantitative methods for literature reviews. Ann Rev Psychol. 2001;52:59-82.

Received for publication: 22 July 2008 